\title{
Bootstrap multiscale analysis and localization for multi-particle continuous Anderson Hamiltonians
}

\begin{abstract}
Abel Klein ${ }^{1}$ and Son T. Nguyen
Abstract. We extend the bootstrap multiscale analysis developed by Germinet and Klein to the multi-particle continuous Anderson Hamiltonian, obtaining Anderson localization with finite multiplicity of eigenvalues, decay of eigenfunction correlations, and a strong form of dynamical localization. We do not require a covering condition. The initial step for this multiscale analysis, required to hold for energies in a nontrivial interval at the bottom of the spectrum, is verified for multi-particle continuous Anderson Hamiltonians. We also extend the unique continuation principle for spectral projections of Schrödinger operators to arbitrary rectangles, and use it to prove Wegner estimates for multi-particle continuous Anderson Hamiltonians without the requirement of a covering condition.
\end{abstract}

Mathematics Subject Classification (2010). Primary 82B44; Secondary 47B80, 60H25, $81 \mathrm{Q} 10$.

Keywords. Multi-particle localization, multi-particle Anderson Hamiltonian, continuous Anderson Hamiltonian, multi-particle random Schrödinger operators, multiscale analysis, Anderson localization, dynamical localization, multi-particle Wegner estimates.

\section{Contents}

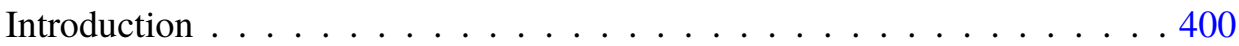

1 Main definitions and results $\ldots \ldots \ldots . \ldots . \ldots 402$

2 Wegner estimates . . . . . . . . . . . . . . . . 409

3 Toolkit for the multiscale analysis . . . . . . . . . . . . 414

4 The initial step for the bootstrap multiscale analysis . . . . . . . . . . 421

5 The multi-particle bootstrap multiscale analysis . . . . . . . . . . . . . 424

6 From the bootstrap multiscale analysis to localization . . . . . . . . 434

A The almost-sure spectrum of the $n$-particle Anderson Hamiltonian . . . 435

B Unique continuation principle for spectral projections of Schrödinger operators on arbitrary rectangles . . . . . . . . . . . 435

References. . . . . . . . . . . . . . . . . . . 441

${ }^{1}$ Abel Klein was supported in part by the NSF under grant DMS-1301641. 


\section{Introduction}

The multi-particle Anderson Hamiltonian is an alloy-type random Schrödinger operator describing $n$ interacting electrons moving in a medium with random impurities. It is the continuous version of the (discrete) multi-particle Anderson model.

Localization was proved for the multi-particle discrete Anderson model by Chulaevsky and Suhov [7, 8, 9], using a multiscale analysis, and Aizenman and Warzel [2], using the fractional moment method. Chulaevsky, Boutet de Monvel and Suhov [6] extended the results of Chulaevsky and Suhov to the multi-particle continuous Anderson Hamiltonian, establishing Anderson and dynamical localization at the bottom of the spectrum.

The bootstrap multiscale analysis, developed in the one-particle case by Germinet and Klein [13] (see also [23]), is an enhanced multiscale analysis that yields sub-exponentially decaying probabilities for 'bad' events. The initial step for the bootstrap multiscale analysis only requires the verification of polynomial decay of the finite volume resolvent, at some sufficiently large scale, with probability bigger than some minimal probability $1-p_{0}$, where $0<p_{0}<1$ is independent of the scale. An important feature of the bootstrap multiscale analysis is that the final probability estimates are independent of the probability estimate in the initial step: any desired sub-exponential decay for the probabilities of 'bad' events can be achieved. The bootstrap multiscale analysis yields Anderson localization with finite multiplicity of eigenvalues, decay of eigenfunction correlations, and a strong form of dynamical localization.

We previously extended the bootstrap multiscale analysis to the multi-particle (discrete) Anderson model [27]. The initial step for the bootstrap multiscale analysis of [27, Theorem 1.5] has to hold for all energies in the spectrum (and hence for all energies); it can be verified for the multi-particle Anderson model at high disorder, as discussed in [27, Remark 1.6].

In this article we extend the bootstrap multiscale analysis and its consequences to the multi-particle (continuous) Anderson Hamiltonian; we do not require a covering condition. The initial step is only required to hold for all energies in a nontrivial interval at the bottom of the spectrum (or equivalently, for all energies below some fixed energy). We also show that we always have this initial step in some nontrivial interval at the bottom of the spectrum for multi-particle Anderson Hamiltonians. The consequences to the bootstrap multiscale analysis include, in addition to Anderson and dynamical localization, new results for multi-particle (continuous) Anderson Hamiltonians: finite multiplicity of eigenvalues, decay of eigenfunction correlations, and a strong form of dynamical localization (see Theorem 1.2). 
Although the results in this paper are written for the continuous multi-particle Anderson Hamiltonian, they also apply to the discrete multi-particle Anderson model, yielding localization at the bottom of the spectrum for the discrete model at any disorder.

The main definitions and results are stated in Section 1. Theorem 1.2 states that continuous multi-particle Anderson Hamiltonians exhibit Anderson localization with finite multiplicity of eigenvalues, decay of eigenfunction correlations, and a strong form of dynamical localization in an interval at the bottom of the spectrum. Theorem 1.6 is the bootstrap multiscale analysis. The consequences regarding localization (Anderson localization with finite multiplicity of eigenvalues, dynamical localization, decay of eigenfunction correlations) are given in Corollary 1.7. In Section 4 we show that the hypotheses of Theorem 1.6 (the initial step for the bootstrap multiscale analysis) are always satisfied at some nontrivial interval at the bottom of the spectrum. Section 3 contains a collection of technical results necessary for the multiscale analysis in the continuum. The proof of Theorem 1.6 is given in Section 5, and the derivation of Corollary 1.7 is discussed in Section 6.

In the multi-particle case events based on disjoint boxes are not necessarily independent, even if the boxes are far apart from each other. This difficulty is overcome by the use of the concepts of partially and fully separated boxes (Subsection 2.1) and partially and fully interactive boxes (Subsection 3.3) introduced by Chulaevsky and Suhov [7, 8, 9]. The relevant distance between boxes is the Hausdorff distance (see (1.6)), introduced in this context by Aizenman and Warzel [2]. In the multiscale analysis partially interactive boxes are handled by the induction hypothesis, i.e., by the conclusions of Theorem 1.6 for a smaller number of particles (see Lemmas 3.9 and 5.1), and fully interactive boxes are handled similarly to one particle boxes (see Lemma 3.11).

The multiscale analysis requires Wegner estimates. Wegner estimates were previously proved for the $n$-particle discrete Anderson model [7, 21, 27]. In the continuum, Wegner estimates for the $n$-particle Anderson Hamiltonian with a covering condition were proved in [28, 3], and without the covering condition in [19].

The one-particle energy interval multiscale analysis $[12,11,13,23]$ requires a two-volume Wegner estimate, i.e., an estimate of the probability of the spectra of independent finite volume Hamiltonians being close together. Chulaevsky and Suhov $[7,8,9]$ realized that for $n$-particles this estimate is required for partially separated finite volume Hamiltonians, that is, finite volume Hamiltonians on partially separated rectangles (here we need rectangles, not just boxes), and proved such an estimate in the discrete case (see also [27]). In the continuum, such an estimate was proved for the $n$-particle Anderson Hamiltonian with a covering con- 
dition [3]. This two-volume Wegner estimate is now proven without the covering condition in [19] and in Corollary 2.3 below by somewhat different arguments.

Our definition of the finite volume random potential (see (1.9)), as well as our definition of fully and partially separated rectangles (Definition 2.1), are slightly different than the ones used in $[3,19]$. While $[3,19]$ take the finite volume random potential to be the restriction of the infinite volume random potential to the $n$-particle rectangle, our finite volume random potential contains only random variables indexed by sites located in the faces of the rectangle (see (1.10)). We prove a Wegner estimate in Theorem 2.2 in which the expectation is taken only with respect to the random variables indexed by one face of the rectangle (a oneparticle box). In Corollary 2.3 we derive from Theorem 2.2 a two-volume Wegner estimate for partially separated rectangles as in Definition 2.1. To do this, in Appendix B we extend the results of [24], proving a unique continuation principle for spectral projections of Schrödinger operators on arbitrary rectangles.

\section{Main definitions and results}

We start by defining the multi-particle Anderson Hamiltonian. We write

$$
\boldsymbol{a}=\left(a_{1}, \ldots, a_{n}\right) \in \mathbb{R}^{n d} \cong\left(\mathbb{R}^{d}\right)^{n},
$$

and set

$$
\|\boldsymbol{a}\|:=\max \left\{\left\|a_{1}\right\|, \ldots,\left\|a_{n}\right\|\right\}
$$

where

$$
\|x\|=\|x\|_{\infty}:=\max \left\{\left|x_{1}\right|, \ldots,\left|x_{d}\right|\right\}
$$

for $x=\left(x_{1}, \ldots, x_{d}\right) \in \mathbb{R}^{d}$.

Definition 1.1. For all $n \in \mathbb{N}$, the $n$-particle Anderson Hamiltonian is the random Schrödinger operator on $L^{2}\left(\mathbb{R}^{n d}\right)$ given by

$$
H_{\omega}^{(n)}:=H_{0, \omega}^{(n)}+U, \quad \text { with } H_{0, \omega}^{(n)}:=-\Delta^{(n)}+V_{\omega}^{(n)},
$$

where we used the following notations. 
(i) $\Delta^{(n)}$ is the $n d$-dimensional Laplacian operator.

(ii) $V_{\boldsymbol{\omega}}^{(n)}$ is the random potential given by $\left(\boldsymbol{x}=\left(x_{1}, \ldots, x_{n}\right) \in \mathbb{R}^{n d}\right)$

$$
V_{\boldsymbol{\omega}}^{(n)}(\boldsymbol{x})=\sum_{i=1, \ldots, n} V_{\boldsymbol{\omega}}^{(1)}\left(x_{i}\right), \quad \text { with } V_{\boldsymbol{\omega}}^{(1)}(x)=\sum_{k \in \mathbb{Z}^{d}} \omega_{k} u(x-k),
$$

where

(a) $\boldsymbol{\omega}=\left\{\omega_{k}\right\}_{k \in \mathbb{Z}^{d}}$ is a family of independent identically distributed random variables whose common probability distribution $\mu$ has a bounded density $\rho$ and satisfies $\left\{0, M_{+}\right\} \subset \operatorname{supp} \mu \subseteq\left[0, M_{+}\right]$for some $M_{+}>0$;

(b) the single site potential $u$ is a measurable function on $\mathbb{R}^{d}$ with

$$
\begin{gathered}
u_{-} \chi_{\Lambda_{\delta_{-}}(0)} \leq u \leq \chi_{\Lambda_{\delta_{+}}(0)} \text { for some constants } u_{-}, \delta_{ \pm} \in(0, \infty) \\
\left(\Lambda_{\delta_{ \pm}}(0)=\left(-\frac{\delta_{ \pm}}{2}, \frac{\delta_{ \pm}}{2}\right)^{d} . \text { We take } u \leq 1 \text { without loss of generality. }\right)
\end{gathered}
$$

(iii) $U$ is a potential governing the finite range interaction between the $n$ particles. We take

$$
U(\boldsymbol{x})=\sum_{1 \leq i<j \leq n} \tilde{U}\left(x_{i}-x_{j}\right),
$$

where $\tilde{U}: \mathbb{R}^{d} \rightarrow[0, \infty)$ is a bounded measurable function, $\widetilde{U}(y)=\widetilde{U}(-y)$, with $\widetilde{U}(y)=0$ for $\|y\|>r_{0}$ for some $0<r_{0}<\infty$.

Remarks. (i) The results of this paper are valid if we only assume that the probability measure $\mu$ is uniformly Hölder continuous, i.e., there exist constants $C<\infty$ and $\alpha \in(0,1]$ such $\mu([a, a+t]) \leq C t^{\alpha}$ for all $a \in \mathbb{R}$ and $t \geq 0$. We assumed that $\mu$ has a bounded density (i.e., $\mu$ is uniformly Hölder continuous with $\alpha=1$ ) for simplicity.

(ii) We took $U$ to be a two-particle interaction potential as in (1.3) for simplicity. Our results hold for nonnegative bounded finite range $n$-particle interaction potentials.

The $n$-particle Anderson Hamiltonian $H_{\omega}^{(n)}$ is a $\mathbb{Z}^{d}$-ergodic random Schrödinger operator on $L^{2}\left(\mathbb{R}^{n d}\right)$. Here $\mathbb{Z}^{d}$ acts on $\mathbb{R}^{n d}$ by

$$
\left(x_{1}, x_{2} \ldots, x_{n}\right) \in \mathbb{R}^{n d} \longmapsto\left(x_{1}+a, x_{2}+a, \ldots, x_{n}+a\right) \in \mathbb{R}^{n d} \text { for } a \in \mathbb{Z}^{d} .
$$

It follows (see [5, Proposition V.2.4]) that there exists fixed subsets $\Sigma^{(n)}, \Sigma_{\mathrm{pp}}^{(n)}$, $\Sigma_{\mathrm{ac}}^{(n)}$ and $\Sigma_{\mathrm{sc}}^{(n)}$ of $\mathbb{R}$ so that the spectrum $\sigma\left(H_{\omega}^{(n)}\right)$ of $H_{\omega}^{(n)}$, as well as its pure point, absolutely continuous, and singular continuous components, are equal to these fixed sets with probability one. 
Note that $H_{\omega}^{(1)}=H_{0, \omega}^{(1)}$, and it is well known that $\Sigma^{(1)}=[0, \infty)($ e.g., [22]). It follows, letting $\Sigma_{0}^{(n)}$ denote the almost sure spectrum of $H_{0, \omega}^{(n)}$, that

$$
\Sigma_{0}^{(n)}=\overline{\Sigma^{(1)}+\cdots+\Sigma^{(1)}}=[0, \infty) .
$$

In Appendix A we show that we also have

$$
\Sigma^{(n)}=[0, \infty)
$$

We now fix a multi-particle Anderson Hamiltonian $H_{\omega}^{(n)}, n \in \mathbb{N}$.

We use the following definitions and notation.

(i) Given $\boldsymbol{a}=\left(a_{1}, \ldots, a_{n}\right) \in \mathbb{R}^{\text {nd }}$, we let

$$
\begin{aligned}
\langle\boldsymbol{a}\rangle & :=\left(1+\|\boldsymbol{a}\|^{2}\right)^{\frac{1}{2}}, \\
\operatorname{diam} \boldsymbol{a} & :=\max _{i, j=1, \ldots, n}\left\|a_{i}-a_{j}\right\|,
\end{aligned}
$$

and

$$
\mathcal{S}_{\boldsymbol{a}}=\left\{a_{1}, \ldots, a_{n}\right\}
$$

(ii) Given $\boldsymbol{a}, \boldsymbol{b} \in \mathbb{R}^{n d}$, we set

$$
d_{H}(\boldsymbol{a}, \boldsymbol{b}):=d_{H}\left(\mathcal{S}_{\boldsymbol{a}}, \mathcal{S}_{\boldsymbol{b}}\right),
$$

where $d_{H}\left(S_{1}, S_{2}\right)$ denotes the Hausdorff distance between two finite subsets $S_{1}, S_{2} \subseteq \mathbb{R}^{d}$, given by

$$
\begin{aligned}
d_{H}\left(S_{1}, S_{2}\right) & :=\max \left\{\max _{x \in S_{1}} \min _{y \in S_{2}}\|x-y\|, \max _{y \in S_{2}} \min _{x \in S_{1}}\|x-y\|\right\} \\
& =\max \left\{\max _{x \in S_{1}} \operatorname{dist}\left(x, S_{2}\right), \max _{y \in S_{2}} \operatorname{dist}\left(y, S_{1}\right)\right\} .
\end{aligned}
$$

It follows from the definition that

$$
d_{H}(\boldsymbol{a}, \boldsymbol{b}) \leq\|\boldsymbol{a}-\boldsymbol{b}\| \leq d_{H}(\boldsymbol{a}, \boldsymbol{b})+\operatorname{diam} \boldsymbol{a} \quad \text { for } \boldsymbol{a}, \boldsymbol{b} \in \mathbb{R}^{n d}
$$

(see [2]).

(iii) We fix $v_{n}>\frac{n d}{2}$ and let $T_{n}$ be the operator on $L^{2}\left(\mathbb{R}^{n d}\right)$ given by multiplication of the function $\langle\boldsymbol{x}\rangle^{v_{n}}$, where $\langle\boldsymbol{x}\rangle=\left(1+\|\boldsymbol{x}\|^{2}\right)^{\frac{1}{2}}$.

(iv) We set

$$
\chi_{\boldsymbol{x}}=\chi_{\left\{\boldsymbol{y} \in \mathbb{R}^{n d} ;\|\boldsymbol{y}-\boldsymbol{x}\|<\frac{1}{2}\right\}} \text { for } \boldsymbol{x} \in \mathbb{R}^{n d} .
$$


We prove localization for the multi-particle Anderson Hamiltonian $H_{\omega}^{(n)}$, for all $n \in \mathbb{N}$, as follows. (Note that $\chi_{\left[0, E_{N}\right)}\left(H_{\omega}^{(N)}\right)=\chi_{\left(-\infty, E_{N}\right)}\left(H_{\omega}^{(N)}\right)$ since $H_{\omega}^{(N)} \geq 0$.)

Theorem 1.2. Given $N \in \mathbb{N}$, there exists an energy $E_{N}>0$ satisfying the following properties.

(i) The following holds with probability one.

(a) Anderson Localization

$H_{\omega}^{(N)}$ has pure point spectrum in the interval $\left[0, E_{N}\right)$. Moreover, there exists $M=M_{N}>0$ such that for all $E \in\left[0, E_{N}\right)$ and $\psi \in \chi_{\{E\}}\left(H_{\omega}^{N}\right)$ we have

$$
\left\|\chi_{\boldsymbol{x}} \psi\right\| \leq C_{\boldsymbol{\omega}, E}\left\|T_{N}^{-1} \psi\right\| e^{-M\|\boldsymbol{x}\|} \quad \text { for all } \boldsymbol{x} \in \mathbb{R}^{N d} .
$$

In particular, each eigenfunction $\psi$ of $H_{\omega}^{(N)}$ with eigenvalue $E \in\left[0, E_{N}\right)$ is exponentially localized with the non-random rate of decay $M>0$.

(b) Finite multiplicity of eigenvalues

The eigenvalues of $H_{\omega}^{(N)}$ in $\left[0, E_{N}\right)$ have finite multiplicity:

$$
\operatorname{tr} \chi_{\{E\}}\left(H_{\omega}^{(N)}\right)<\infty \text { for all } E \in\left[0, E_{N}\right) .
$$

(c) Summable uniform decay of eigenfunction CORRelaTIONS (SUDEC)

For every $\zeta \in(0,1)$ there exists a constant $C_{\omega, \zeta}$ such that for every $E \in\left[0, E_{N}\right)$ and $\phi, \psi \in \operatorname{Ran} \chi_{\{E\}}\left(H_{\omega}^{(N)}\right)$ we have

$$
\left\|\chi_{\boldsymbol{x}} \phi\right\|\left\|\chi_{\boldsymbol{y}} \psi\right\| \leq C_{\boldsymbol{\omega}, \zeta}\left\|T_{N}^{-1} \phi\right\|\left\|T_{N}^{-1} \psi\right\|\langle\boldsymbol{x}\rangle^{2 v} e^{-\left(d_{H}(\boldsymbol{x}, \boldsymbol{y})\right)^{\zeta}}
$$

for all $\boldsymbol{x}, \boldsymbol{y} \in \mathbb{R}^{N d}$.

(ii) Dynamical Localization

For every $\zeta \in(0,1)$ and $\boldsymbol{y} \in \mathbb{R}^{N d}$ there exists a constant $C_{\zeta}(\boldsymbol{y})$ such that

$$
\mathbb{E}\left\{\sup _{|g| \leq 1}\left\|\chi_{\boldsymbol{x}} \chi_{\left[0, E_{N}\right)}\left(H_{\boldsymbol{\omega}}^{(N)}\right) g\left(H_{\boldsymbol{\omega}}^{(N)}\right) \chi_{\boldsymbol{y}}\right\|\right\} \leq C_{\zeta}(\boldsymbol{y}) e^{-\left(d_{H}(\boldsymbol{x}, \boldsymbol{y})\right)^{\zeta}}
$$

for all $\boldsymbol{x}, \boldsymbol{y} \in \mathbb{R}^{N d}$, the supremum being taken over Borel functions $g$ on $\mathbb{R}$ with $\sup _{t \in \mathbb{R}}|g(t)| \leq 1$. In particular, we have

$$
\mathbb{E}\left\{\sup _{t \in \mathbb{R}}\left\|\chi_{\boldsymbol{x}} \chi_{\left[0, E_{N}\right)}\left(H_{\boldsymbol{\omega}}^{(N)}\right) e^{i t H_{\boldsymbol{\omega}}^{(N)}} \chi_{\boldsymbol{y}}\right\|\right\} \leq C_{\zeta}(\boldsymbol{y}) e^{-\left(d_{H}(\boldsymbol{x}, \boldsymbol{y})\right)^{\zeta}}
$$

for all $\boldsymbol{x} \in \mathbb{R}^{N d}$. 
Remark. SUDEC (Summable Uniform Decay of Eigenfunction Correlations) is equivalent to SULE (Semi Uniformly Localized Eigenfunctions); see [17, Remark 3].

The theorem is proved by a bootstrap multiscale analysis, a statement about finite volume multi-particle Anderson Hamiltonians. Our finite volumes will be boxes and rectangles, defined as follows.

(i) The one-particle box centered at $x \in \mathbb{R}^{d}$ with side of length $L>0$ is $\Lambda_{L}(x)=\left\{y \in \mathbb{R}^{d} ;\|y-x\|<\frac{L}{2}\right\}$. We set

$$
\hat{\Lambda}=\Lambda \cap \mathbb{Z}^{d} \text {. }
$$

(ii) The $n$-particle box centered at $x \in \mathbb{R}^{n d}$ with side length $L>0$ is

$$
\boldsymbol{\Lambda}_{L}^{(n)}(\boldsymbol{x})=\left\{\boldsymbol{y} \in \mathbb{R}^{n d} ;\|\boldsymbol{y}-\boldsymbol{x}\|<\frac{L}{2}\right\}=\prod_{i=1}^{n} \Lambda_{L}\left(x_{i}\right) ;
$$

note that $\Lambda_{L}^{(1)}(x)=\Lambda_{L}(x)$. By a box $\Lambda_{L}$ in $\mathbb{R}^{n d}$ we mean an $n$-particle box $\boldsymbol{\Lambda}_{L}^{(n)}(\boldsymbol{x})$ for some $\boldsymbol{x} \in \mathbb{R}^{n d}$. Note that $\chi_{\boldsymbol{x}}=\chi_{\boldsymbol{\Lambda}_{1}(\boldsymbol{x})}$ for $\boldsymbol{x} \in \mathbb{R}^{n d}$.

(iii) We also define $n$-particle rectangles in $\mathbb{R}^{n d}$ centered at points $\boldsymbol{x} \in \mathbb{R}^{n d}$ :

$$
\boldsymbol{\Lambda}^{(n)}(\boldsymbol{x})=\prod_{i=1}^{n} \Lambda_{L_{i}}\left(x_{i}\right), \quad \text { where } L_{1}, L_{2}, \ldots, L_{n}>0 .
$$

(We mostly use $n$-particle boxes, but in a few places we will need $n$-particle rectangles.)

Definition 1.3. Given an $n$-particle rectangle

$$
\boldsymbol{\Lambda}=\boldsymbol{\Lambda}^{(n)}(\boldsymbol{a})=\prod_{i=1}^{n} \Lambda_{L_{i}}\left(a_{i}\right)
$$

we define the corresponding finite volume Anderson Hamiltonian $H_{\boldsymbol{\omega}, \boldsymbol{\Lambda}}^{(n)}$ on $\mathrm{L}^{2}(\boldsymbol{\Lambda})$ by

$$
H_{\boldsymbol{\omega}, \boldsymbol{\Lambda}}^{(n)}:=H_{0, \boldsymbol{\omega}, \boldsymbol{\Lambda}}^{(n)}+U_{\boldsymbol{\Lambda}}, \quad \text { with } \quad H_{0, \boldsymbol{\omega}, \boldsymbol{\Lambda}}^{(n)}:=-\Delta_{\boldsymbol{\Lambda}}^{(n)}+V_{\boldsymbol{\omega}, \boldsymbol{\Lambda}}^{(n)},
$$

where $\Delta_{\boldsymbol{\Lambda}}^{(n)}$ is the Laplacian on $\boldsymbol{\Lambda}$ with Dirichlet boundary condition, $U_{\boldsymbol{\Lambda}}$ is the restriction of $U$ to $\boldsymbol{\Lambda}$, and

$$
V_{\boldsymbol{\omega}, \boldsymbol{\Lambda}}^{(n)}(\boldsymbol{x})=\sum_{i=1}^{n} V_{\boldsymbol{\omega}, \Lambda_{L_{i}}\left(a_{i}\right)}^{(1)}\left(x_{i}\right) \quad \text { for } \boldsymbol{x} \in \mathbf{\Lambda},
$$


where $V_{\omega, \Lambda}^{(1)}$ is defined for a one-particle box $\Lambda \subseteq \mathbb{R}^{d}$ by

$$
V_{\omega, \Lambda}^{(1)}(x)=\sum_{k \in \hat{\Lambda}} \omega_{k} u(x-k) \quad \text { for } x \in \Lambda .
$$

We set

$$
R_{\boldsymbol{\omega}, \mathbf{\Lambda}}^{(n)}(z)=\left(H_{\boldsymbol{\omega}, \boldsymbol{\Lambda}}^{(n)}-z\right)^{-1} \quad \text { for } z \notin \sigma\left(H_{\boldsymbol{\omega}, \mathbf{\Lambda}}^{(n)}\right) .
$$

Note that $H_{\omega, \Lambda}^{(1)}=H_{0, \omega, \Lambda}^{(1)}$ and we have (cf. (1.4))

$$
\sigma\left(H_{0, \boldsymbol{\omega}, \mathbf{\Lambda}}^{(n)}\right)=\sigma\left(H_{\boldsymbol{\omega}, \Lambda_{L_{1}}\left(a_{1}\right)}^{(1)}\right)+\cdots+\sigma\left(H_{\boldsymbol{\omega}, \Lambda_{L_{n}}\left(a_{n}\right)}^{(1)}\right) .
$$

We will often omit the dependency on $n$ from the notation, where it is clear, and just write $H_{\boldsymbol{\omega}, \boldsymbol{\Lambda}}$ for $H_{\boldsymbol{\omega}, \boldsymbol{\Lambda}}^{(n)}$ and $R_{\boldsymbol{\omega}, \boldsymbol{\Lambda}}(z)$ for $R_{\boldsymbol{\omega}, \boldsymbol{\Lambda}}^{(n)}(z)$.

The bootstrap multiscale analysis uses three types of good boxes, defined for a fixed $\omega$ (omitted from the notation).

Definition 1.4. Let $\boldsymbol{\Lambda}=\boldsymbol{\Lambda}_{L}^{(n)}(\boldsymbol{x})$ be an $n$-particle box and let $E \in \mathbb{R}$. Let $\theta>0$, $\zeta \in(0,1)$, and $m>0$.

(i) The $n$-particle box $\boldsymbol{\Lambda}$ is $(\theta, E)$-suitable if, and only if, $E \notin \sigma\left(H_{\boldsymbol{\Lambda}}\right)$ and

$$
\left\|\chi_{\boldsymbol{a}} R_{\boldsymbol{\Lambda}}(E) \chi_{\boldsymbol{b}}\right\| \leq L^{-\theta} \quad \text { for all } \boldsymbol{a}, \boldsymbol{b} \in \boldsymbol{\Lambda} \text { with }\|\boldsymbol{a}-\boldsymbol{b}\| \geq \frac{L}{100} .
$$

Otherwise, $\boldsymbol{\Lambda}$ is called $(\theta, E)$-nonsuitable.

(ii) The $n$-particle box $\boldsymbol{\Lambda}$ is ( $\zeta, E)$-subexponentially suitable (SES) if, and only if, $E \notin \sigma\left(H_{\boldsymbol{\Lambda}}\right)$ and

$$
\left\|\chi_{\boldsymbol{a}} R_{\boldsymbol{\Lambda}}(E) \chi_{\boldsymbol{b}}\right\| \leq e^{-L^{\zeta}} \quad \text { for all } \boldsymbol{a}, \boldsymbol{b} \in \boldsymbol{\Lambda} \text { with }\|\boldsymbol{a}-\boldsymbol{b}\| \geq \frac{L}{100} .
$$

Otherwise, $\boldsymbol{\Lambda}$ is called ( $\zeta, E$ )-nonsubexponentially suitable (nonSES).

(iii) The $n$-particle box $\boldsymbol{\Lambda}$ is (m,E)-regular if, and only if, $E \notin \sigma\left(H_{\boldsymbol{\Lambda}}\right)$ and

$$
\left\|\chi_{\boldsymbol{a}} R_{\boldsymbol{\Lambda}}(E) \chi_{\boldsymbol{b}}\right\| \leq e^{-m\|\boldsymbol{a}-\boldsymbol{b}\|} \quad \text { for all } \boldsymbol{a}, \boldsymbol{b} \in \boldsymbol{\Lambda} \quad \text { with }\|\boldsymbol{a}-\boldsymbol{b}\| \geq \frac{L}{100} .
$$

Otherwise, $\boldsymbol{\Lambda}$ is called ( $m, E)$-nonregular. 
Remark 1.5. The different types of good boxes are related:

(i) $\boldsymbol{\Lambda}_{L}^{(n)}(\boldsymbol{x})(m, E)$-regular $\Longrightarrow \boldsymbol{\Lambda}_{L}^{(n)}(\boldsymbol{x})\left(\frac{m L}{100 \log L}, E\right)$-suitable;

(ii) $\boldsymbol{\Lambda}_{L}^{(n)}(\boldsymbol{x})(\theta, E)$-suitable $\Longrightarrow \boldsymbol{\Lambda}_{L}^{(n)}(\boldsymbol{x})\left(\frac{\theta \log L}{L}, E\right)$-regular;

(iii) $\Lambda_{L}^{(n)}(\boldsymbol{x})\left(L^{\zeta-1}, E\right)$-regular $\Longrightarrow \Lambda_{L}^{(n)}(\boldsymbol{x})\left(\zeta-\frac{\log 100}{\log L}, E\right)-\mathrm{SES}$;

(iv) $\Lambda_{L}^{(n)}(\boldsymbol{x})(\zeta, E)-\mathrm{SES} \Longrightarrow \boldsymbol{\Lambda}_{L}^{(n)}(\boldsymbol{x})\left(L^{\zeta-1}, E\right)$-regular.

Our main technical result extends the bootstrap multiscale analysis of Germinet and Klein [13] (see also [23]) to the multi-particle Anderson Hamiltonian.

Theorem 1.6 (bootstrap multiscale analysis). There exist

$$
p_{0}(n)=p_{0}(d, n)>0, \quad n=1,2, \ldots,
$$

such that, for every $N \in \mathbb{N}$, given $\theta>8 N d$ and an energy $E^{(N)}>0$, there exists

$$
\mathcal{L}=\mathcal{L}\left(d,\|\rho\|_{\infty}, N, \theta, E^{(N)}\right)
$$

such that if, for some $L_{0} \geq \mathcal{L}$ and all $n=1,2, \ldots, N$,

$$
\sup _{\boldsymbol{x} \in \mathbb{R}^{n d}} \mathbb{P}\left\{\boldsymbol{\Lambda}_{L_{0}}^{(n)}(\boldsymbol{x}) \text { is }(\theta, E) \text {-nonsuitable }\right\} \leq p_{0}(n)
$$

for all

$$
E \leq E^{(n)}:=2^{N-n} E^{(N)},
$$

then, given $0<\zeta<1$, we can find a length scale

$$
\begin{gathered}
L_{\zeta}=L_{\zeta}\left(d,\|\rho\|_{\infty}, N, \theta, E^{(N)}, L_{0}\right), \\
\delta_{\zeta}=\delta_{\zeta}\left(d,\|\rho\|_{\infty}, N, \theta, E^{(N)}, L_{0}\right)>0,
\end{gathered}
$$

and

$$
m_{\zeta}=m_{\zeta}\left(\delta_{\zeta}, L_{\zeta}\right)>0
$$

so that the following holds for $n=1,2, \ldots, N$. 
(i) For every $E \leq E^{(n)}, L \geq L_{\zeta}$, and $\boldsymbol{a} \in \mathbb{R}^{n d}$,

$$
\mathbb{P}\left\{\boldsymbol{\Lambda}_{L}^{(n)}(\boldsymbol{a}) \text { is }\left(m_{\zeta}, E\right) \text {-nonregular }\right\} \leq e^{-L^{\zeta}} .
$$

(ii) Given $E_{1}<E^{(n)}$, set

$$
I\left(E_{1}\right)=\left[E_{1}-\delta_{\zeta}, E_{1}+\delta_{\zeta}\right] \cap\left(-\infty, E^{(n)}\right] .
$$

Then, for every $E_{1}<E^{(n)}, L \geq L_{\zeta}$, and $\boldsymbol{a}, \boldsymbol{b} \in \mathbb{R}^{n d}$ with $d_{H}(\boldsymbol{a}, \boldsymbol{b}) \geq L$,

$\mathbb{P}\left\{\right.$ there exists $E \in I\left(E_{1}\right)$ such that

$$
\left.\boldsymbol{\Lambda}_{L}^{(n)}(\boldsymbol{a}) \text { and } \boldsymbol{\Lambda}_{L}^{(n)}(\boldsymbol{b}) \text { are }\left(m_{\zeta}, E\right) \text {-nonregular }\right\} \leq e^{-L^{\zeta}} .
$$

Theorem 4.1 shows that the hypotheses of Theorem 1.6 are always satisfied at some nontrivial interval at the the bottom of the spectrum.

Corollary 1.7 (localization). Given $N \in \mathbb{N}$, an energy $E^{(N)}>0$, and an open interval $I \subseteq\left(-\infty, E^{(N)}\right)$, suppose that the conclusions of Theorem 1.6 hold for all energies $E \in I$. Then the conclusions of Theorem 1.2 hold on the interval I (i.e., with I substituted for the interval $\left[0, E_{N}\right)$ in Theorem 1.2).

Theorem 1.2 follows immediately from Theorem 1.6, Theorem 4.1, and Corollary 1.7.

\section{Wegner estimates}

2.1. Fully and partially separated rectangles. Let

$$
\boldsymbol{\Lambda}=\boldsymbol{\Lambda}^{(n)}(\boldsymbol{a})=\prod_{i=1}^{n} \Lambda_{L_{i}}\left(a_{i}\right)
$$

be an $n$-particle rectangle. Given $\mathcal{J} \subseteq\{1, \ldots, n\}$, we set

$$
\begin{gathered}
\boldsymbol{\Lambda}\left(\boldsymbol{a}_{\mathfrak{J}}\right)=\boldsymbol{\Lambda}^{\mathcal{J}}\left(\boldsymbol{a}_{\mathcal{J}}\right)=\prod_{i \in \mathcal{J}} \Lambda_{L_{i}}\left(a_{i}\right), \quad \text { where } \boldsymbol{a}_{\mathcal{J}}=\left(a_{i}, i \in \mathcal{J}\right), \boldsymbol{a}=\left(\boldsymbol{a}_{\mathfrak{J}}, \boldsymbol{a}_{\mathfrak{J}}\right) ; \\
\Pi_{\mathcal{J}} \boldsymbol{\Lambda}^{(n)}(\boldsymbol{a})=\bigcup_{i \in \mathcal{J}} \Lambda_{L_{i}}\left(a_{i}\right), \quad \Pi_{i} \boldsymbol{\Lambda}^{(n)}(\boldsymbol{a})=\Pi_{\{i\}} \boldsymbol{\Lambda}^{(n)}(\boldsymbol{a})=\Lambda_{L_{i}}\left(a_{i}\right) ; \\
\Pi_{\boldsymbol{\Lambda}}^{(n)}(\boldsymbol{a})=\Pi_{\{1, \ldots, n\}} \boldsymbol{\Lambda}^{(n)}(\boldsymbol{a}) .
\end{gathered}
$$


Definition 2.1. Let

$$
\boldsymbol{\Lambda}^{(n)}(\boldsymbol{x})=\prod_{i=1}^{n} \Lambda_{L_{i}}\left(x_{i}\right) \quad \text { and } \quad \boldsymbol{\Lambda}^{(n)}(\boldsymbol{y})=\prod_{i=1}^{n} \Lambda_{\ell_{i}}\left(y_{i}\right)
$$

be a pair of $n$-particle rectangles.

(i) $\boldsymbol{\Lambda}^{(n)}(\boldsymbol{x})$ and $\boldsymbol{\Lambda}^{(n)}(\boldsymbol{y})$ are partially separated if, and only if, either

$$
\Lambda_{L_{i}}\left(x_{i}\right) \cap \Pi \Lambda^{(n)}(y)=\emptyset \quad \text { for some } i \in\{1, \ldots, n\},
$$

or

$$
\Lambda_{\ell_{j}}\left(y_{j}\right) \cap \Pi \Lambda^{(n)}(\boldsymbol{x})=\emptyset \quad \text { for some } j \in\{1, \ldots, n\}
$$

(ii) $\boldsymbol{\Lambda}^{(n)}(\boldsymbol{x})$ and $\boldsymbol{\Lambda}^{(n)}(\boldsymbol{y})$ are fully separated if, and only if,

$$
\Pi \Lambda^{(n)}(\boldsymbol{x}) \cap \Pi \Lambda^{(n)}(\boldsymbol{y})=\emptyset .
$$

Note that, in view of our definition of the finite volume random potentials (see (1.9) and (1.10)), events based on fully separated rectangles are independent. Moreover, if the $n$-particle rectangles $\boldsymbol{\Lambda}^{(n)}(\boldsymbol{x})$ and $\boldsymbol{\Lambda}^{(n)}(\boldsymbol{y})$ are partially separated, with, say, $\Lambda_{L_{i}}\left(x_{i}\right) \cap \Pi \boldsymbol{\Lambda}^{(n)}(\boldsymbol{y})=\emptyset$, then events based on $\boldsymbol{\Lambda}^{(n)}(\boldsymbol{y})$ are independent of the random variables $\left\{\omega_{k} ; k \in \widehat{\Lambda_{L_{i}}\left(x_{i}\right)}\right\}$.

2.2. The Wegner estimates. Given a one-particle box $\Lambda_{L}(x)$, we will use $\mathbb{E}_{\Lambda_{L}(x)}$ and $\mathrm{P}_{\Lambda_{L}(x)}$ to denote the expectation and probability with respect to the probability distribution of the random variables $\left\{\omega_{k} ; k \in \widehat{\Lambda_{L}(x)}\right\}$.

Theorem 2.2. Let $n \in \mathbb{N}$ and $E_{+}>0$. There exist constants

$$
\gamma_{n, E_{+}}=\gamma_{n, E_{+}}\left(d, M_{+}, \delta_{-},\|\tilde{U}\|_{\infty}\right)>0
$$

and

$$
C_{n, E_{+}}=C\left(d, M_{+}, u_{-}, \delta_{ \pm},\|\tilde{U}\|_{\infty}, n, E_{+}\right),
$$

such that, for all n-particle rectangles

$$
\boldsymbol{\Lambda}=\boldsymbol{\Lambda}^{(n)}(\boldsymbol{a})=\prod_{i=1}^{n} \Lambda_{L_{i}}\left(a_{i}\right)
$$

with $\boldsymbol{a}=\left(a_{1}, \ldots, a_{n}\right) \in \mathbb{R}^{n d}$ and $114 \sqrt{n d} \leq L_{i} \leq L$ for $i=1, \ldots, n$, and all intervals $I \subseteq\left[0, E_{+}\right)$with $|I| \leq 2 \gamma_{n, E_{+}}$, we have

$$
\mathbb{E}_{\Lambda_{L_{i}}\left(a_{i}\right)}\left\{\operatorname{tr} \chi_{I}\left(H_{\boldsymbol{\omega}, \mathbf{\Lambda}}^{(n)}\right)\right\} \leq C_{n, E_{+}}\|\rho\|_{\infty}|I| L^{n d} \quad \text { for } i=1,2, \ldots, n .
$$


In particular, for any $E \leq E_{+}, 0<\varepsilon \leq \gamma_{n, E_{+}}$, and $i=1,2, \ldots, n$, we have

$$
\begin{aligned}
\mathbb{P}_{\Lambda_{L_{i}}\left(a_{i}\right)}\left\{\left\|R_{\omega, \boldsymbol{\Lambda}}^{(n)}(E)\right\| \geq \frac{1}{\varepsilon}\right\} & =\mathbb{P}_{\Lambda_{L_{i}}\left(a_{i}\right)}\left\{d\left(\sigma\left(H_{\omega, \boldsymbol{\Lambda}}^{(n)}\right), E\right) \leq \varepsilon\right\} \\
& \leq 2 C_{n, E_{+}}\|\rho\|_{\infty} \varepsilon L^{n d} .
\end{aligned}
$$

We prove Theorem 2.2 by modifying the proof of [19, Theorem 1]. The main difference between Theorem 2.2 and [19, Theorem 1] is that the expectation in (2.2) is taken only with respect to the random variables indexed by the one-particle box $\Lambda_{q}$. This is needed for proving Corollary 2.3 for a pair of partially separated $n$-particle rectangles. Note also that Theorem 2.2 is proved for arbitrary $n$-particle rectangles, not just $n$-particle boxes $\Lambda_{L}^{(n)}(\boldsymbol{a})$ with $\boldsymbol{a} \in \mathbb{Z}^{n d}$ and $L \in \mathbb{N}$ as in [19, Theorem 1] - a consequence of their use of the results of [24]. We extend the results of [24] to arbitrary $n$-particle rectangles in Appendix B.

Proof. Let

$$
\boldsymbol{\Lambda}=\prod_{i=1, \ldots, n} \Lambda_{i}, \quad \text { where } \Lambda_{i}=\Lambda_{L_{i}}\left(a_{i}\right)
$$

be an $n$-particle rectangle with $\boldsymbol{a} \in \mathbb{R}^{n d}$ and $114 \sqrt{n d} \leq L_{i} \leq L$ for $i=1, \ldots, n$. Then for $\boldsymbol{x} \in \Lambda$ we have

$$
\begin{aligned}
V_{\boldsymbol{\omega}, \boldsymbol{\Lambda}}^{(n)}(\boldsymbol{x}) & =\sum_{i=1}^{n} V_{\boldsymbol{\omega}, \Lambda_{i}}^{(1)}\left(x_{i}\right) \\
& =\sum_{i=1}^{n} \sum_{k \in \widehat{\Lambda}_{i}} \omega_{k} u\left(x_{i}-k\right) \\
& =\sum_{k \in \mathbb{Z}^{d}} \omega_{k}\left(\sum_{i ; k \in \widehat{\Lambda_{i}}} u\left(x_{i}-k\right)\right) \\
& =\sum_{k \in \mathbb{Z}^{d}} \omega_{k} \theta_{k}^{(\boldsymbol{\Lambda})}(\boldsymbol{x}),
\end{aligned}
$$

where

$$
\theta_{k}^{(\boldsymbol{\Lambda})}(\boldsymbol{x})=\sum_{\left\{i ; k \in \widehat{\Lambda}_{i}\right\}} u\left(x_{i}-k\right) \geq u_{-} \sum_{\left\{i ; k \in \widehat{\Lambda}_{i}\right\}} \chi_{\Lambda_{\delta-}^{(1)}(k)}\left(x_{i}\right),
$$

where we used (1.2). It follows that for $q=1,2, \ldots, n$ we have

$$
\begin{aligned}
H_{\boldsymbol{\omega}, \boldsymbol{\Lambda}}^{(n)} & =-\Delta_{\boldsymbol{\Lambda}}^{(n)}+U_{\boldsymbol{\Lambda}}+\sum_{k \in \mathbb{Z}^{d}} \omega_{k} \theta_{k}^{(\boldsymbol{\Lambda})} \\
& =-\Delta_{\boldsymbol{\Lambda}}^{(n)}+U_{\boldsymbol{\Lambda}}+\sum_{k \in \mathbb{Z}^{d} \backslash \widehat{\Lambda_{q}}} \omega_{k} \theta_{k}^{(\mathbf{\Lambda})}+\sum_{k \in \widehat{\Lambda_{q}}} \omega_{k} \theta_{k}^{(\mathbf{\Lambda})} .
\end{aligned}
$$


Now, for

$$
\eta=\min \left\{\frac{\delta_{-}}{2}, \frac{1}{2}\right\} \quad \text { and } \quad B_{\eta}^{(n)}(\boldsymbol{k})=\left\{\boldsymbol{x} \in \mathbb{R}^{n d} ;|\boldsymbol{x}-\boldsymbol{k}|_{2}<\eta\right\},
$$

we define

$$
W^{(\boldsymbol{\Lambda})}(\boldsymbol{x})=\sum_{\boldsymbol{k} \in \mathbf{\Lambda} \cap \mathbb{Z}^{n d}} \chi_{B_{\eta}^{(n)}(\boldsymbol{k})}(\boldsymbol{x}) \text { for } \boldsymbol{x} \in \boldsymbol{\Lambda} .
$$

Fix $q \in\{1, \ldots, n\}$, and given $\boldsymbol{x} \in \mathbb{R}^{n d}$, write

$$
\boldsymbol{x}=\left(x_{q}, \boldsymbol{x}_{q}^{\perp}\right), \quad \text { where } \boldsymbol{x}_{q}^{\perp} \in \mathbb{R}^{(n-1) d} .
$$

Then

$$
\chi_{B_{\eta}^{(n)}(\boldsymbol{k})}(\boldsymbol{x}) \leq \chi_{\boldsymbol{B}_{\eta}^{(1)}\left(k_{q}\right)}\left(x_{q}\right) \chi_{\boldsymbol{B}_{\eta}^{(n-1)}\left(\boldsymbol{k}_{q}^{\perp}\right)}\left(\boldsymbol{x}_{q}^{\perp}\right) \quad \text { for } \boldsymbol{k} \in \mathbb{Z}^{n d}, \boldsymbol{x} \in \mathbb{R}^{n d} .
$$

We write

$$
\boldsymbol{\Lambda}=\Lambda_{q} \times \boldsymbol{\Lambda}_{q}^{\perp}, \quad \text { where } \boldsymbol{\Lambda}_{q}^{\perp}=\prod_{i \in\{1, \ldots, n\} \backslash\{q\}} \Lambda_{i} .
$$

It follows that, for all $\boldsymbol{x} \in \boldsymbol{\Lambda}$,

$$
\begin{aligned}
W^{(\boldsymbol{\Lambda})}(\boldsymbol{x}) & \leq \sum_{\boldsymbol{k} \in \boldsymbol{\Lambda} \cap \mathbb{Z}^{n d}} \chi_{B_{\eta}^{(1)}\left(k_{q}\right)}\left(x_{q}\right) \chi_{B_{\eta}^{(n-1)}\left(\boldsymbol{k}_{q}^{\perp}\right)}\left(\boldsymbol{x}_{q}^{\perp}\right) \\
& =\sum_{k_{q} \in \widehat{\Lambda_{q}}} \chi_{B_{\eta}^{(1)}\left(k_{q}\right)}\left(x_{q}\right)\left\{\sum_{\boldsymbol{k}_{q}^{\perp} \in \boldsymbol{\Lambda}}^{\perp \cap \cap \mathbb{Z}^{(n-1) d}} \chi_{B_{\eta}^{(n-1)}\left(\boldsymbol{k}_{q}^{\perp}\right)}\left(\boldsymbol{x}_{q}^{\perp}\right)\right\} \\
& \leq \sum_{k_{q} \in \widehat{\Lambda_{q}}} \chi_{B_{\eta}^{(1)}\left(k_{q}\right)}\left(x_{q}\right) \\
& \leq \sum_{k \in \widehat{\Lambda_{q}}} \chi_{\Lambda_{\delta-}^{(1)}(k)}\left(x_{q}\right) \\
& \left.\leq \sum_{k \in \widehat{\Lambda_{q}}} \sum_{i ; k \in \widehat{\Lambda_{i}}} \chi_{\Lambda_{\delta-}^{(1)}(k)}\left(x_{i}\right)\right) \\
& \leq u_{-}^{-1} \sum_{k \in \widehat{\Lambda_{q}}} \theta_{k}^{(\boldsymbol{\Lambda})}(\boldsymbol{x}) .
\end{aligned}
$$

Fix $E_{+}>0$. It follows from Theorem B.1 that for any interval $I \subseteq\left[0, E_{+}\right)$ with $|I| \leq 2 \gamma_{n, E_{+}}$,

$$
\begin{aligned}
\chi_{I}\left(H_{\boldsymbol{\omega}, \boldsymbol{\Lambda}}^{(n)}\right) & \leq \gamma_{n, E_{+}}^{-2} \chi_{I}\left(H_{\boldsymbol{\omega}, \boldsymbol{\Lambda}}^{(n)}\right) W^{(\Lambda)} \chi_{I}\left(H_{\boldsymbol{\omega}, \boldsymbol{\Lambda}}^{(n)}\right) \\
& \leq u_{-}^{-1} \gamma_{n, E_{+}}^{-2} \chi_{I}\left(H_{\boldsymbol{\omega}, \boldsymbol{\Lambda}}^{(n)}\right)\left(\sum_{k \in \widehat{\Lambda_{q}}} \theta_{k}^{(\boldsymbol{\Lambda})}\right) \chi_{I}\left(H_{\boldsymbol{\omega}, \boldsymbol{\Lambda}}^{(n)}\right),
\end{aligned}
$$


for all $\omega \in\left[0, M_{+}\right]^{\mathbb{Z}^{d}}$, where $\gamma_{n, E_{+}}$is obtained from (B.5):

$$
\gamma_{n, E_{+}}^{2}=\frac{1}{2} \eta^{M_{n d}\left(1+K^{\frac{2}{3}}\right)} \quad \text { with } K=n(n-1)\|\widetilde{U}\|_{\infty}+2 M_{+} \delta_{+}^{d}+E_{+} .
$$

Wegner estimate (2.2) can be proved following the strategy of [24, Lemma 3.1], using (2.4) and (2.6). This is what is done in [19, Proof of Theorem 1], the difference being that the proof in [19] uses a version of (2.6) where in the right hand side

$$
\sum_{k \in \widehat{\Lambda_{q}}} \theta_{k}^{(\boldsymbol{\Lambda})} \quad \text { is replaced by } \sum_{q=1}^{d} \sum_{k \in \widehat{\Lambda_{q}}} \theta_{k}^{(\boldsymbol{\Lambda})},
$$

and averages over all random variables instead of only over the random variables $\left\{\omega_{i}\right\}_{i \in \widehat{\Lambda_{q}}}$. The same argument as in [19] applies, using (2.6) and averaging only over the random variables $\left\{\omega_{i}\right\}_{i \in \widehat{\Lambda_{q}}}$, yielding (2.2).

Corollary 2.3. Let $n \in \mathbb{N}$ and $E_{+}>0$, and let $\gamma_{n, E_{+}}$be as in Theorem 2.2. Let

$$
\boldsymbol{\Lambda}_{1}=\prod_{i=1, \ldots, n} \Lambda_{L_{i}}\left(a_{i}\right) \quad \text { and } \quad \boldsymbol{\Lambda}_{2}=\prod_{i=1, \ldots, n} \Lambda_{L_{i}^{\prime}}\left(b_{i}\right)
$$

with $\boldsymbol{a}, \boldsymbol{b} \in \mathbb{R}^{n d}$ and $114 \sqrt{n d} \leq L_{i}, L_{i}^{\prime} \leq L$ for $i=1, \ldots, n$, be a pair of partially separated n-particle rectangles. Set

$$
\tilde{\sigma}\left(H_{\boldsymbol{\Lambda}_{1}}\right)=\sigma\left(H_{\boldsymbol{\Lambda}_{1}}\right) \cap\left(-\infty, E_{+}\right],
$$

and

$$
\tilde{\sigma}\left(H_{\boldsymbol{\Lambda}_{2}}\right)=\sigma\left(H_{\boldsymbol{\Lambda}_{2}}\right) \cap\left(-\infty, E_{+}\right]
$$

Then there exists a constant

$$
\widetilde{C}_{n, E_{+}}=\tilde{C}\left(d, M_{+}, u_{-}, \delta_{ \pm},\|\tilde{U}\|_{\infty}, n, E_{+}\right),
$$

such that, for all $0<\varepsilon \leq \gamma_{n, E_{+}}$,

$$
\mathbb{P}\left\{\operatorname{dist}\left(\tilde{\sigma}\left(H_{\boldsymbol{\Lambda}_{1}}\right), \tilde{\sigma}\left(H_{\boldsymbol{\Lambda}_{2}}\right)\right) \leq \varepsilon\right\} \leq \widetilde{C}_{n, E_{+}}\|\rho\|_{\infty} \varepsilon L^{2 n d} .
$$

Corollary 2.3 follows from Theorem 2.2 in the same way [27, Corollary 2.4] is derived from [27, Theorem 2.3]. Note that Corollary 2.3 is (up to minor details) the same as [19, Theorem 9], although the proofs use somewhat different versions of the Wegner estimate. 


\section{Toolkit for the multiscale analysis}

3.1. Deterministic estimates. The following lemmas are deterministic, i.e., they hold for a fixed $\omega$ (omitted from the notation).

Given $n$-particle boxes $\boldsymbol{\Lambda} \subseteq \tilde{\boldsymbol{\Lambda}}$ we set

$$
\begin{aligned}
& \partial^{\tilde{\mathbf{\Lambda}} \boldsymbol{\Lambda}}:=\partial \boldsymbol{\Lambda} \backslash \partial \tilde{\boldsymbol{\Lambda}}, \\
& \boldsymbol{\Lambda}^{\tilde{\mathbf{\Lambda}}, \delta}:=\left\{\boldsymbol{x} \in \boldsymbol{\Lambda} ; \operatorname{dist}\left(\boldsymbol{x}, \partial^{\tilde{\boldsymbol{\Lambda}}} \boldsymbol{\Lambda}\right) \geq \delta\right\} \quad \text { for } \delta>0, \\
& \Upsilon=\Upsilon_{\boldsymbol{\Lambda}}^{\tilde{\Lambda}}:=\left\{\boldsymbol{x} \in \boldsymbol{\Lambda} ; \operatorname{dist}\left(\boldsymbol{x}, \partial^{\tilde{\Lambda}} \boldsymbol{\Lambda}\right)=\frac{1+\delta_{+}}{2}=: \tilde{\delta}_{+}\right\}, \\
& \tilde{\Upsilon}=\tilde{\Upsilon}_{\Lambda}^{\tilde{\Lambda}}:=\bigcup_{x \in \Upsilon}\left(\Lambda_{\frac{1}{2}}(x) \cap \Lambda\right) .
\end{aligned}
$$

Lemma 3.1. Let $\boldsymbol{\Lambda} \subsetneq \tilde{\boldsymbol{\Lambda}}$ be two $n$-particles boxes of length $\ell$ and $L$, respectively, with $\ell<L$, and $z \notin \sigma\left(H_{\mathbf{\Lambda}}\right) \cup \sigma\left(H_{\tilde{\mathbf{\Lambda}}}\right)$. Then there exists a constant

$$
C=C_{n, d},
$$

such that, for $\boldsymbol{x} \in \boldsymbol{\Lambda}$ with $\boldsymbol{\Lambda}_{3+\delta_{+}}(\boldsymbol{x}) \cap \tilde{\boldsymbol{\Lambda}} \subseteq \boldsymbol{\Lambda}$, and $\boldsymbol{y} \in \tilde{\boldsymbol{\Lambda}} \backslash \boldsymbol{\Lambda}$, we can find $\boldsymbol{a} \in \Upsilon_{\boldsymbol{\Lambda}}^{\tilde{\boldsymbol{\Lambda}}}$ such that

$$
\left\|\chi_{\boldsymbol{y}} R_{\widetilde{\mathbf{\Lambda}}}(z) \chi_{\boldsymbol{x}}\right\| \leq C_{n, d} \ell^{n d-1} \sqrt{5+\max \{0, \Re(z)\}}\left\|\chi_{\boldsymbol{y}} R_{\widetilde{\mathbf{\Lambda}}}(z) \chi_{\boldsymbol{a}}\right\|\left\|\chi_{\boldsymbol{a}} R_{\boldsymbol{\Lambda}}(z) \chi_{\boldsymbol{x}}\right\| .
$$

In particular, if $\Re(z) \leq E^{(n)}$, for some fixed energy $E^{(n)}$, we get

$$
\left\|\chi_{\boldsymbol{y}} R_{\widetilde{\mathbf{\Lambda}}}(z) \chi_{\boldsymbol{x}}\right\| \leq \ell^{n d}\left\|\chi_{\boldsymbol{y}} R_{\widetilde{\mathbf{\Lambda}}}(z) \chi_{\boldsymbol{a}}\right\|\left\|\chi_{\boldsymbol{a}} R_{\mathbf{\Lambda}}(z) \chi_{\boldsymbol{x}}\right\|,
$$

provided $\ell$ is sufficiently large (depending on $E^{(n)}$ ).

Lemma 3.1 is just [18, Lemma 2.4(i)] with minor modifications.

Lemma 3.2. Given an $n$-particle box, $\boldsymbol{\Lambda}$ of side $\ell$, for every $E \geq 0$ we have

$$
\#\left\{\lambda \in \sigma\left(H_{\boldsymbol{\Lambda}}^{(n)}\right) \cap(-\infty, E]\right\}=\operatorname{tr}\left\{\chi_{(-\infty, E]}\left(H_{\boldsymbol{\Lambda}}^{(n)}\right)\right\} \leq C_{n d} E^{\frac{n d}{2}} \ell^{n d} .
$$

Lemma 3.2 follows from [25, Lemma 3.3] (see also [16, Eq. (A.7)]).

We also use the following Combes-Thomas estimate from [14, Eq. (19) in Theorem 1]. 
Lemma 3.3. Let $\boldsymbol{\Lambda}$ be an n-particle box. Then for all $E<\inf \sigma\left(H_{\boldsymbol{\Lambda}}\right)$ we have

$$
\left\|\chi_{\boldsymbol{x}} R_{\boldsymbol{\Lambda}}(E) \chi_{\boldsymbol{y}}\right\| \leq \frac{4}{3}\left(\inf \sigma\left(H_{\boldsymbol{\Lambda}}\right)-E\right)^{-1} e^{-\frac{1}{2} \sqrt{\inf \sigma\left(H_{\boldsymbol{\Lambda}}\right)-E}(\|\boldsymbol{x}-\boldsymbol{y}\|-n d)}
$$

for all $\boldsymbol{x}, \boldsymbol{y} \in \mathbb{R}^{\text {nd }}$. In particular, if we take $\|\boldsymbol{x}-\boldsymbol{y}\| \geq \frac{L}{100}$ with L sufficiently large, we have

$$
\begin{aligned}
\left\|\chi_{\boldsymbol{x}} R_{\boldsymbol{\Lambda}}(E) \chi_{\boldsymbol{y}}\right\| & \leq \frac{4}{3}\left(\inf \sigma\left(H_{\boldsymbol{\Lambda}}\right)-E\right)^{-1} e^{-\frac{1}{3} \sqrt{\inf \sigma\left(H_{\boldsymbol{\Lambda}}\right)-E}\|\boldsymbol{x}-\boldsymbol{y}\|} \\
& \leq \frac{4}{3}\left(\inf \sigma\left(H_{\boldsymbol{\Lambda}}\right)-E\right)^{-1} e^{-\frac{\sqrt{\inf \sigma\left(H_{\boldsymbol{\Lambda}}\right)-E}}{300} L} .
\end{aligned}
$$

3.2. Suitable cover. Following [18, Definition 3.12] we introduce suitable covers of $n$-particle boxes.

Definition 3.4. Given scales $\ell<L$, a suitable $\ell$-covering of a box $\Lambda_{L}^{(N)}(\boldsymbol{x})$ is a collection of boxes $\boldsymbol{\Lambda}_{\ell}$ of the form

$$
\mathcal{G}_{\boldsymbol{\Lambda}_{L}^{(\ell)}(\boldsymbol{x})}^{(\ell)}=\left\{\boldsymbol{\Lambda}_{\ell}(\boldsymbol{r})\right\}_{\boldsymbol{r} \in \mathbb{G}_{\boldsymbol{\Lambda}_{L}^{(N)}(\boldsymbol{x})}^{(\ell)}},
$$

where

$$
\mathbb{G}_{\boldsymbol{\Lambda}_{L}^{(N)}(x)}^{(x)}:=\left\{\boldsymbol{x}+\alpha \ell \mathbb{Z}^{N d}\right\} \cap \boldsymbol{\Lambda}_{L}^{(N)}(\boldsymbol{x}) \quad \text { with } \alpha \in\left[\frac{3}{5}, \frac{4}{5}\right] \cap\left\{\frac{L-\ell}{2 \ell n} ; n \in \mathbb{N}\right\} .
$$

Suitable covers are useful because of [18, Lemma 3.13], stated below.

Lemma 3.5. Let $\ell \leq \frac{L}{6}$. Then every box $\Lambda_{L}^{(N)}(x)$ has a suitable $\ell$-covering, and for any suitable l-covering $\mathcal{G}_{\boldsymbol{\Lambda}_{L}^{(\ell)}(\boldsymbol{x})}^{(N)}$ of $\boldsymbol{\Lambda}_{L}^{(N)}(\boldsymbol{x})$,

$$
\boldsymbol{\Lambda}_{L}^{(N)}(\boldsymbol{x})=\bigcup_{\boldsymbol{r} \in \mathbb{G}_{\boldsymbol{\Lambda}_{L}^{(\ell)}(N)}^{(x)}} \boldsymbol{\Lambda}_{\ell}(\boldsymbol{r}) ;
$$

for each $\boldsymbol{y} \in \boldsymbol{\Lambda}_{L}^{(N)}(\boldsymbol{x})$ there is $\boldsymbol{r} \in \mathbb{G}_{\boldsymbol{\Lambda}_{L}^{(N)}(\boldsymbol{x})}^{(\ell)}$ such that

$$
\begin{gathered}
\boldsymbol{\Lambda}_{\frac{\ell}{5}}(y) \cap \boldsymbol{\Lambda}_{L}^{(N)}(\boldsymbol{x}) \subset \boldsymbol{\Lambda}_{\ell}(\boldsymbol{r}) ; \\
\boldsymbol{\Lambda}_{\frac{\ell}{5}}(\boldsymbol{r}) \cap \boldsymbol{\Lambda}_{\ell}\left(\boldsymbol{r}^{\prime}\right)=\emptyset \quad \text { for all } \boldsymbol{r}, \boldsymbol{r}^{\prime} \in \boldsymbol{x}+\alpha \ell \mathbb{Z}^{d}, \boldsymbol{r} \neq \boldsymbol{r}^{\prime} \\
\left(\frac{L}{\ell}\right)^{N d} \leq \# \mathbb{G}_{\boldsymbol{\Lambda}_{L}^{(N)}(\boldsymbol{x})}^{(\ell)}=\left(\frac{L-\ell}{\alpha \ell}+1\right)^{N d} \leq\left(\frac{2 L}{\ell}\right)^{N d} .
\end{gathered}
$$


Moreover, given $\boldsymbol{y} \in \boldsymbol{x}+\alpha \ell \mathbb{Z}^{N d}$ and $k \in \mathbb{N}$, it follows that

$$
\boldsymbol{\Lambda}_{(2 k \alpha+1) \ell}(\boldsymbol{y})=\bigcup_{\boldsymbol{r} \in\left\{\boldsymbol{x}+\alpha \ell \mathbb{Z}^{N d}\right\} \cap \boldsymbol{\Lambda}_{(2 k \alpha+1) \ell}(\boldsymbol{y})} \boldsymbol{\Lambda}_{\ell}(\boldsymbol{r}),
$$

and $\left\{\boldsymbol{\Lambda}_{\ell}(\boldsymbol{r})\right\}_{\boldsymbol{r} \in\left\{\boldsymbol{x}+\alpha \ell \mathbb{Z}^{N d}\right\} \cap \boldsymbol{\Lambda}_{(2 k \alpha+1) \ell}(\boldsymbol{y})}$ is a suitable $\ell$-covering of $\boldsymbol{\Lambda}_{(2 k \alpha+1) \ell}(\boldsymbol{y})$. In particular, for each $\boldsymbol{y} \in \mathbb{Z}^{N d}$ there is $\boldsymbol{r} \in \boldsymbol{x}+\alpha \ell \mathbb{Z}^{N d}$ such that

$$
\boldsymbol{\Lambda}_{\frac{\ell}{5}}(\boldsymbol{y}) \subset \boldsymbol{\Lambda}_{\ell}(\boldsymbol{r})
$$

Remark 3.6. In performing the $N$-particle multiscale analysis, we will utilize Lemma 3.5 in the following way: we first choose some appropriate $k_{1}$ such that $2 k_{1} \alpha+1>3 N$. Given $J \in \mathbb{N}$, let $\boldsymbol{a}_{1}, \cdots, \boldsymbol{a}_{t} \in \boldsymbol{\Lambda}_{L}^{(N)}(\boldsymbol{x})$ where $t \leq J N^{N}$, there exists

$$
\boldsymbol{y}_{1}, \ldots, \boldsymbol{y}_{t} \in \boldsymbol{x}+\alpha \ell \mathbb{Z}^{N d}
$$

such that

$$
\begin{aligned}
\boldsymbol{\Lambda}_{3 N \ell}\left(\boldsymbol{a}_{1}\right) \cap \boldsymbol{\Lambda}_{L}^{(N)}(\boldsymbol{x}) & \subseteq \boldsymbol{\Lambda}_{\left(2 k_{1} \alpha+1\right) \ell}\left(\boldsymbol{y}_{1}\right) \\
& \subseteq \boldsymbol{\Lambda}_{L}^{(N)}(\boldsymbol{x}), \ldots, \boldsymbol{\Lambda}_{3 N \ell}\left(\boldsymbol{a}_{t}\right) \cap \boldsymbol{\Lambda}_{L}^{(N)}(\boldsymbol{x}) \\
& \subseteq \boldsymbol{\Lambda}_{\left(2 k_{1} \alpha+1\right) \ell}\left(\boldsymbol{y}_{t}\right) \\
& \subseteq \boldsymbol{\Lambda}_{L}^{(N)}(\boldsymbol{x}) .
\end{aligned}
$$

If the set

$$
\boldsymbol{\Lambda}_{\left(2 k_{1} \alpha+1\right) \ell}\left(\boldsymbol{y}^{(1)}\right) \cup \cdots \cup \boldsymbol{\Lambda}_{\left(2 k_{1} \alpha+1\right) \ell}\left(\boldsymbol{y}^{(m)}\right) \subseteq \boldsymbol{\Lambda}_{L}^{(N)}(\boldsymbol{x}),
$$

where

$$
\boldsymbol{y}^{(1)}, \ldots, \boldsymbol{y}^{(m)} \in \boldsymbol{x}+\alpha \ell \mathbb{Z}^{N d}
$$

is connected, then we will take $k_{m}$ to be the smallest integer such that

$$
\boldsymbol{\Lambda}_{\left(2 k_{1} \alpha+1\right) \ell}\left(\boldsymbol{y}^{(1)}\right) \cup \cdots \cup \boldsymbol{\Lambda}_{\left(2 k_{1} \alpha+1\right) \ell}\left(\boldsymbol{y}^{(m)}\right) \subseteq \boldsymbol{\Lambda}_{\left(2 k_{m} \alpha+1\right) \ell}(\boldsymbol{r}) \subseteq \boldsymbol{\Lambda}_{L}^{(N)}(\boldsymbol{x}),
$$

for some $\boldsymbol{r} \in \boldsymbol{x}+\alpha \ell \mathbb{Z}^{N d}$. Moreover, for each $\boldsymbol{y} \in \mathbb{Z}^{N d}$, we set

$$
\Lambda_{\ell}^{(y)}=\Lambda_{\ell}(\boldsymbol{r})
$$

where $\boldsymbol{\Lambda}_{\ell}(\boldsymbol{r})$ comes from (3.13). 
3.3. Partially and fully interactive boxes. Following Chulaevsky and Suhov $[8,9]$, we divide $n$-particle boxes into two types: partially interactive and fully interactive.

Definition 3.7. An $n$-particle box $\Lambda_{L}^{(n)}(\boldsymbol{a})$ is partially interactive (PI) when there exists $\emptyset \neq \mathcal{J} \subsetneq\{1, \ldots, n\}$ such that $\Lambda_{L}^{(n)}(a) \subseteq \mathcal{E}_{\mathcal{J}}$, where

$$
\mathcal{E}_{\mathfrak{J}}=\left\{\boldsymbol{x} \in \mathbb{R}^{n d} \mid \min _{i \in \mathcal{J}, j \notin \mathcal{J}}\left\|x_{i}-x_{j}\right\|>r_{0}\right\} .
$$

If $\Lambda_{L}^{(n)}(\boldsymbol{a})$ is not partially interactive, then it is said fully interactive (FI).

If the $n$-particle box $\Lambda_{L}^{(n)}(\boldsymbol{a})$ is partially interactive, by writing

$$
\Lambda_{L}^{(n)}(a)=\Lambda_{L}^{\mathcal{J}}\left(\mathbf{a}_{\mathfrak{\jmath}}\right) \times \Lambda_{L}^{\jmath^{c}}\left(\mathbf{a}_{\mathfrak{\jmath} c}\right)
$$

we are implicitly stating that

$$
\Lambda_{L}^{(n)}(a) \subseteq \mathcal{E}_{\mathcal{J}} \quad \text { for } \emptyset \neq \mathcal{J} \subsetneq\{1, \ldots, n\} .
$$

We set

$$
\sigma_{\mathfrak{J}}=\sigma\left(H_{\boldsymbol{\Lambda}_{L}^{\mathfrak{g}}\left(\mathbf{a}_{\mathfrak{f}}\right)}\right) \quad \text { and } \quad \sigma_{\mathfrak{J} c}=\sigma\left(H_{\boldsymbol{\Lambda}_{L}^{\mathfrak{g}}\left(\mathbf{a}_{\mathfrak{f} c}\right)}\right) .
$$

Given $\lambda \in \sigma_{\mathfrak{\jmath}}$, we write

$$
P_{\lambda}^{\mathcal{J}}=\chi_{\{\lambda\}}\left(H_{\mathbf{\Lambda}_{L}^{\mathcal{J}}\left(\mathbf{a}_{\jmath}\right)}\right)
$$

Lemma 3.8. Let

$$
\boldsymbol{\Lambda}_{L}^{(n)}(\boldsymbol{u})=\boldsymbol{\Lambda}_{L}^{\mathcal{J}}\left(\mathbf{u}_{\mathfrak{\jmath}}\right) \times \boldsymbol{\Lambda}_{L}^{\mathfrak{f}^{c}}\left(\mathbf{u}_{\mathfrak{\jmath} c}\right)
$$

be a PI n-particle box. Then

(i) $\Pi_{\jmath} \boldsymbol{\Lambda}_{L}^{(n)}(\boldsymbol{u}) \bigcap \Pi_{\mathfrak{\gamma} c} \boldsymbol{\Lambda}_{L}^{(n)}(\boldsymbol{u})=\emptyset$, so events based on $\boldsymbol{\Lambda}_{L}^{\mathcal{J}}\left(\mathbf{u}_{\mathcal{J}}\right)$ and $\boldsymbol{\Lambda}_{L}^{\mathfrak{\jmath} c}\left(\mathbf{u}_{\mathfrak{\jmath} c}\right)$ are independent;

(ii) $H_{\boldsymbol{\Lambda}_{L}^{(n)}(\boldsymbol{u})}=H_{L} H_{\mathcal{J}}\left(\mathbf{u}_{\mathfrak{f})} \otimes I_{L} I_{\left(\mathbf{u}_{\mathfrak{f} c}\right)}+I_{L}^{I_{\mathcal{J}}\left(\mathbf{u}_{\mathfrak{f}}\right)} \otimes H_{L} H_{\left(\mathbf{u}_{\mathfrak{f} c}\right)} ;\right.$

(iii) $\sigma\left(H_{\boldsymbol{\Lambda}_{L}^{(n)}(\boldsymbol{u})}\right)=\sigma\left(H_{\boldsymbol{\Lambda}_{L}\left(\mathbf{u}_{\mathcal{f}}\right)}\right)+\sigma\left(H_{\boldsymbol{\Lambda}_{L}\left(\mathbf{u}_{\mathfrak{g}} \mathbf{c}\right)}\right)$; 
(iv) if $z \notin \sigma\left(H_{\boldsymbol{\Lambda}_{L}^{(n)}(\boldsymbol{u})}\right)$, then

$$
\begin{aligned}
R_{\boldsymbol{\Lambda}_{L}^{(n)}(\boldsymbol{u})}(z) & =\sum_{\lambda \in \sigma_{\mathcal{J}}} \sum_{\mu \in \sigma_{\mathcal{J}} c} \frac{1}{\lambda+\mu-z} P_{\lambda}^{\mathcal{J}} \otimes P_{\mu}^{\mathcal{J}^{c}} \\
& =\sum_{\lambda \in \sigma_{\mathfrak{J}}} P_{\lambda}^{\mathcal{J}} \otimes R_{\boldsymbol{\Lambda}_{L}\left(\mathbf{u}_{\mathfrak{J}} \mathbf{c}\right)}(z-\lambda) \\
& =\sum_{\mu \in \sigma_{\mathfrak{f} c}} R_{\boldsymbol{\Lambda}_{L}\left(\mathbf{u}_{\mathfrak{J}}\right)}(z-\mu) \otimes P_{\mu}^{\mathcal{J}^{c}}
\end{aligned}
$$

and, for all $\boldsymbol{x}, \boldsymbol{y} \in \boldsymbol{\Lambda}_{L}^{(n)}(\boldsymbol{u})$, we get

$$
\left\|\chi_{\boldsymbol{x}} R_{\boldsymbol{\Lambda}}(z) \chi_{\boldsymbol{y}}\right\| \leq \sum_{\lambda \in \sigma_{\mathfrak{J}}}\left\|\chi_{\boldsymbol{x}_{\mathfrak{f} c}} R_{\boldsymbol{\Lambda}_{L}\left(\mathbf{u}_{\mathfrak{f}} \mathbf{c}\right)}(z-\lambda) \chi_{\boldsymbol{y}_{\mathfrak{f} c}}\right\|
$$

and

$$
\left\|\chi_{\boldsymbol{x}} R_{\boldsymbol{\Lambda}}(z) \chi_{\boldsymbol{y}}\right\| \leq \sum_{\mu \in \sigma_{\mathfrak{J}} c}\left\|\chi_{\boldsymbol{x}_{\mathfrak{J}}} R_{\boldsymbol{\Lambda}_{L}\left(\mathbf{u}_{\mathfrak{J}}\right)}(z-\mu) \chi_{\boldsymbol{y}_{\mathfrak{J}}}\right\| .
$$

Lemma 3.9. Let

$$
\boldsymbol{\Lambda}_{\ell}^{(n)}(\boldsymbol{u})=\boldsymbol{\Lambda}_{\ell}^{\mathcal{J}}\left(\mathbf{u}_{\mathcal{J}}\right) \times \boldsymbol{\Lambda}_{\ell}^{\mathcal{J}^{c}}\left(\mathbf{u}_{\mathcal{J} c}\right)
$$

be a PI n-particle box and $E \leq E^{(n)}$. If $\ell$ is sufficiently large, the following holds.

(i) Given $\theta>2 n d+2$, suppose that $\mathbf{\Lambda}_{\ell}^{\mathcal{J}}\left(\mathbf{u}_{\mathfrak{J}}\right)$ is $(\theta, E-\mu)$-suitable for every $\mu \in \sigma_{\mathfrak{g} c} \cap\left(-\infty, 2 E^{(n)}\right]$ and that $\mathbf{\Lambda}_{\ell}^{\mathfrak{g}^{c}}\left(\mathbf{u}_{\mathfrak{g} \mathbf{c}}\right)$ is $(\theta, E-\lambda)$-suitable for every $\lambda \in \sigma_{\mathcal{J}} \cap\left(-\infty, 2 E^{(n)}\right]$. Then $\boldsymbol{\Lambda}_{\ell}^{(n)}(\boldsymbol{u})$ is $\left(\frac{\theta}{2}, E\right)$-suitable.

(ii) Given $0<m \leq \frac{1}{6} \sqrt{E^{(n)}}$, suppose that $\mathbf{\Lambda}_{\ell}^{\mathcal{J}}\left(\mathbf{u}_{\mathcal{J}}\right)$ is $(m, E-\mu)$-regularfor every $\mu \in \sigma_{\mathfrak{g} c} \cap\left(-\infty, 2 E^{(n)}\right]$ and that $\mathbf{\Lambda}_{\ell}^{\mathcal{J}^{c}}\left(\mathbf{u}_{\mathfrak{g} \mathbf{c}}\right)$ is $(m, E-\lambda)$-regular for every $\lambda \in \sigma_{\mathfrak{J}} \cap\left(-\infty, 2 E^{(n)}\right]$. Then $\boldsymbol{\Lambda}_{\ell}^{(n)}(\boldsymbol{u})$ is $\left(m-\frac{100(n d+1) \log (2 \ell)}{\ell}, E\right)$-regular.

(iii) Given $0<\zeta^{\prime}<\zeta<1$, suppose that $\boldsymbol{\Lambda}_{\ell}^{\mathcal{J}}\left(\mathbf{u}_{\mathcal{J}}\right)$ is $(\zeta, E-\mu)$-SES for every $\mu \in \sigma_{\mathfrak{g} c} \cap\left(-\infty, 2 E^{(n)}\right]$ and that $\mathbf{\Lambda}_{\ell}^{\mathfrak{f}^{c}}\left(\mathbf{u}_{\mathfrak{J} \mathbf{c}}\right)$ is $(\zeta, E-\lambda)$-SES for every $\lambda \in \sigma_{\mathcal{J}} \cap\left(-\infty, 2 E^{(n)}\right]$. Then $\boldsymbol{\Lambda}_{\ell}^{(n)}(\boldsymbol{u})$ is $\left(\zeta^{\prime}, E\right)-S E S$.

Proof. We prove (ii), the proofs of (i) and (iii) are similar. Given $\boldsymbol{x}, \boldsymbol{y} \in \boldsymbol{\Lambda}_{\ell}^{(N)}(\boldsymbol{u})$ with $\|x-y\| \geq \frac{\ell}{100}$, then either we have

$$
\left\|\boldsymbol{x}_{\mathfrak{J}}-\boldsymbol{y}_{\mathcal{J}}\right\| \geq \frac{\ell}{100} \quad \text { or } \quad\left\|\boldsymbol{x}_{\mathfrak{\jmath} c}-\boldsymbol{y}_{\mathfrak{g} c}\right\| \geq \frac{\ell}{100} .
$$


Without loss of generality, we suppose that

$$
\left\|\boldsymbol{x}_{\mathfrak{J}}-\boldsymbol{y}_{\mathfrak{J}}\right\| \geq \frac{\ell}{100}
$$

Then, using (3.14), Lemma 3.2, and the fact that $\boldsymbol{\Lambda}_{\mathfrak{\jmath}}=\boldsymbol{\Lambda}_{\ell}^{\mathfrak{\jmath}}\left(\mathbf{u}_{\mathfrak{\jmath}}\right)$ is $(m, E-\mu)$-regular for every $\mu \in \sigma_{\mathcal{J} c} \cap\left(-\infty, 2 E^{(n)}\right]$, and setting

$$
\sigma_{\mathfrak{J} c}(E)=\sigma_{\mathfrak{J} c} \cap(-\infty, E],
$$

we get

$$
\begin{aligned}
& \left\|\chi_{\boldsymbol{x}} R_{\boldsymbol{\Lambda}}(E) \chi_{\boldsymbol{y}}\right\| \\
& \leq \sum_{\mu \in \sigma_{\mathfrak{f} C}}\left\|\chi_{\boldsymbol{x}_{\mathfrak{f}}} R_{\boldsymbol{\Lambda}_{\mathfrak{f}}}(E-\mu) \chi_{\boldsymbol{y}_{\mathfrak{g}}}\right\| \\
& =\sum_{\mu \in \sigma_{\mathfrak{f} c}\left(2 E^{(n)}\right)}\left\|\chi_{\boldsymbol{x}_{\mathfrak{\jmath}}} R_{\boldsymbol{\Lambda}_{\mathfrak{f}}}(E-\mu) \chi_{\boldsymbol{y}_{\mathfrak{\jmath}}}\right\| \\
& +\sum_{\mu \in \sigma_{\mathfrak{f} c} \backslash \sigma_{\mathfrak{f} c}\left(2 E^{(n)}\right)}\left\|\chi_{\boldsymbol{x}_{\mathfrak{\jmath}}} R_{\boldsymbol{\Lambda}_{\mathfrak{\jmath}}}(E-\mu) \chi_{\boldsymbol{y}_{\mathfrak{\jmath}}}\right\| \\
& \leq C_{n d}\left(2 E^{(n)}\right)^{\frac{n d}{2}} \ell^{n d} e^{-m\left\|\boldsymbol{x}_{\mathfrak{J}}-\boldsymbol{y}_{\ni}\right\|} \\
& +\sum_{\mu \in \sigma_{\mathfrak{J} c} \backslash \sigma_{\mathfrak{J} c}\left(2 E^{(n)}\right)}\left\|\chi_{\boldsymbol{x}_{\mathfrak{f}}} R_{\boldsymbol{\Lambda}_{\mathfrak{f}}}(E-\mu) \chi_{\boldsymbol{y}_{\mathfrak{J}}}\right\| \\
& \leq \ell^{n d+1} e^{-m\left\|\boldsymbol{x}_{\mathfrak{\gamma}}-\boldsymbol{y}_{\mathcal{\jmath}}\right\|}+\sum_{k=2}^{\infty} \sum_{\substack{\mu \in \sigma_{\mathcal{J}} c \\
k E^{(n)}<\mu \leq(k+1) E^{(n)}}}\left\|\chi_{\boldsymbol{x}_{\mathfrak{\jmath}}} R_{\boldsymbol{\Lambda}_{\mathfrak{\jmath}}}(E-\mu) \chi_{\boldsymbol{y}_{\mathfrak{\jmath}}}\right\| .
\end{aligned}
$$

Applying (3.5) for $\mu \in \sigma_{\mathcal{J} c}$ with $k E^{(n)}<\mu \leq(k+1) E^{(n)}$ we get

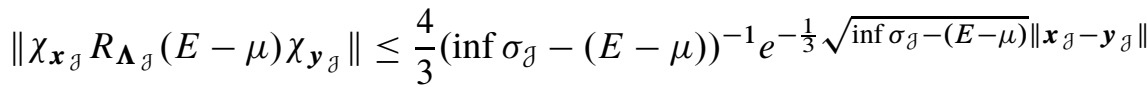

$$
\begin{aligned}
& \leq \frac{4}{3}\left(k E^{(n)}-E\right)^{-1} e^{-\frac{1}{3} \sqrt{k E^{(n)}-E}\left\|\boldsymbol{x}_{\mathfrak{\jmath}}-\boldsymbol{y}_{\text {子 }}\right\|}
\end{aligned}
$$

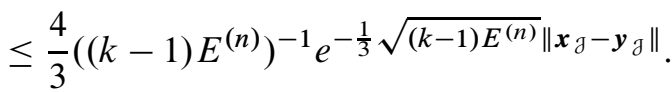


Using Lemma 3.2 and (), we have $\left(k \geq 2,\left\|\boldsymbol{x}_{\mathfrak{J}}-\boldsymbol{y}_{\mathfrak{J}}\right\| \geq \frac{\ell}{100}\right)$

$$
\begin{aligned}
\sum_{\substack{\mu \in \sigma_{\mathfrak{\jmath} c} \\
k E^{(n)}<\mu \leq(k+1) E^{(n)}}}\left\|\chi_{\boldsymbol{x}_{\mathfrak{\jmath}}} R_{\mathbf{\Lambda}_{\mathfrak{\jmath}}}(E-\mu) \chi_{\boldsymbol{y}_{\mathfrak{\jmath}}}\right\| \\
\quad \leq C_{n d} \ell^{n d}\left((k+1) E^{(n)}\right)^{\frac{n d}{2}-1} \mathrm{e}^{-\frac{1}{3} \sqrt{(k-1) E^{(n)}}\left\|\boldsymbol{x}_{\mathfrak{\jmath}}-\boldsymbol{y}_{\mathfrak{\jmath}}\right\|} \\
\leq e^{-\frac{1}{6} \sqrt{(k-1) E^{(n)}}\left\|\boldsymbol{x}_{\mathfrak{\jmath}}-\boldsymbol{y}_{\mathfrak{g}}\right\|},
\end{aligned}
$$

for sufficiently large $\ell$, so

$$
\begin{aligned}
& \sum_{k=2}^{\infty} \sum_{\substack{\mu \in \sigma_{\mathfrak{\jmath} c} \\
k E^{(n)}<\mu \leq(k+1) E^{(n)}}}\left\|\chi_{\boldsymbol{x}_{\mathfrak{\jmath}}} R_{\boldsymbol{\Lambda}_{\mathfrak{\jmath}}}(E-\mu) \chi_{\boldsymbol{y}_{\mathfrak{\jmath}}}\right\| \\
& \leq \sum_{k=2}^{\infty} e^{-\frac{1}{6} \sqrt{(k-1) E^{(n)}}\left\|\boldsymbol{x}_{\mathfrak{\jmath}}-\boldsymbol{y}_{\mathfrak{\jmath}}\right\|} \\
& \leq 2 e^{-\frac{1}{6} \sqrt{E^{(n)}}\left\|\boldsymbol{x}_{\mathfrak{\jmath}}-\boldsymbol{y}_{\mathfrak{g}}\right\|},
\end{aligned}
$$

for $\ell$ large. Using (3.15) and $m \leq \frac{1}{6} \sqrt{E^{(n)}}$, we get

$$
\begin{aligned}
& \left\|\chi_{\boldsymbol{x}} R_{\boldsymbol{\Lambda}}(E) \chi_{\boldsymbol{y}}\right\| \leq \ell^{n d+1} e^{-m\left\|\boldsymbol{x}_{\mathfrak{g}}-\boldsymbol{y}_{\mathcal{J}}\right\|}+2 e^{-\frac{1}{6} \sqrt{E^{(n)}}\left\|\boldsymbol{x}_{\mathfrak{J}}-\boldsymbol{y}_{\mathfrak{g}}\right\|} \\
& \leq 2 \ell^{n d+1} e^{-m\left\|\boldsymbol{x}_{\mathfrak{J}}-\boldsymbol{y}_{\mathfrak{J}}\right\|} \\
& \leq e^{-\left(m-\frac{100(n d+1) \log (2 \ell)}{\ell}\right)\|\boldsymbol{x}-\boldsymbol{y}\|} .
\end{aligned}
$$

Definition 3.10. Let $\Lambda_{L}^{(n)}(\boldsymbol{a})$ and $\boldsymbol{\Lambda}_{L}^{(n)}(\boldsymbol{b})$ be a pair of $n$-particle boxes. We say $\boldsymbol{\Lambda}_{L}^{(n)}(\boldsymbol{a})$ and $\boldsymbol{\Lambda}_{L}^{(n)}(\boldsymbol{b})$ are L-distant when

$$
\max \left\{\operatorname{dist}\left(\boldsymbol{b}, \mathcal{S}_{\boldsymbol{a}}^{n}\right), \operatorname{dist}\left(\boldsymbol{a}, S_{\boldsymbol{b}}^{n}\right)\right\} \geq 3 n L .
$$

The following lemma gives a sufficient condition for a pair of FI $n$-particle boxes to be fully separated, and hence for events based on these boxes to be independent. We omit the proof.

Lemma 3.11. Let $\boldsymbol{\Lambda}_{L}^{(n)}(\boldsymbol{a})$ and $\boldsymbol{\Lambda}_{L}^{(n)}(\boldsymbol{b})$ be a pair of FI n-particle boxes, where $L$ is sufficiently large. Then $\Lambda_{L}^{(n)}(\boldsymbol{a})$ and $\boldsymbol{\Lambda}_{L}^{(n)}(\boldsymbol{b})$ are fully separated if

$$
\max _{x \in \mathcal{S}_{\boldsymbol{a}}, y \in \mathcal{S}_{\boldsymbol{b}}}\|x-y\| \geq 3 n L .
$$

In particular, a pair of L-distant FI n-particle boxes are fully separated. 


\subsection{Resonant rectangles}

Definition 3.12. Let

$$
\Lambda=\prod_{i=1, \ldots, n} \Lambda_{L_{i}}\left(a_{i}\right)
$$

with $L=\min _{i=1, \ldots, n}\left\{L_{i}\right\}>0$ be an $n$-particle rectangle, $E \in \mathbb{R}, s>0$, and $\beta \in(0,1)$.

(i) $\boldsymbol{\Lambda}$ is called $(E, s)$-suitably resonant provided

$$
\operatorname{dist}\left(\sigma\left(H_{\boldsymbol{\Lambda}}^{(n)}\right), E\right)<L^{-s} .
$$

Otherwise, $\boldsymbol{\Lambda}$ is said $(E, s)$-suitably nonresonant.

(ii) $\boldsymbol{\Lambda}$ is called $(E, \beta)$-resonant provided

$$
\operatorname{dist}\left(\sigma\left(H_{\Lambda}^{(n)}\right), E\right)<\frac{1}{2} e^{-L^{\beta}} .
$$

Otherwise, $\boldsymbol{\Lambda}$ is said $(E, \beta)$-nonresonant.

\section{The initial step for the bootstrap multiscale analysis}

We now show that the hypotheses of Theorem 1.6 are verified for energies at the bottom of the spectrum. Recall $\Sigma^{(n)}=[0, \infty)$.

Theorem 4.1. Let $\theta>0$ and $0<p_{0}<1$, and fix $\varepsilon>0$. Then for all $n \in \mathbb{N}$ there exists

$$
\mathcal{L}_{n}=\mathcal{L}_{n}\left(d, u_{-}, \delta_{ \pm}, \mu, \theta, p_{0}, \varepsilon\right),
$$

such that, for all $L \geq \mathcal{L}_{n}$ and $\boldsymbol{x} \in \mathbb{R}^{n d}$,

$$
\mathbb{P}\left\{\boldsymbol{\Lambda}_{L}^{(n)}(\boldsymbol{x}) \text { is }(\theta, E) \text {-suitable }\right\} \geq 1-p_{0} \quad \text { for all } E \leq E_{L}^{(n)},
$$

where

$$
E_{L}^{(n)}=\frac{n}{2}\left(d \log \left(L+\delta_{+}+2\right)-\log p_{0}+\log n\right)^{-\frac{2+\varepsilon}{d}} .
$$

Proof. We start with a well known result for the one-particle case. Fix $\theta>0$, $p_{0}>0$, and $n \in \mathbb{N}, \varepsilon>0$, and set

$$
p_{n}=\frac{p_{0}}{n} .
$$


As shown in [18, Proof of Proposition 4.3], there exists an energy

$$
E_{1}=E_{1}\left(d, u_{-}, \delta_{-}, \mu, \varepsilon\right)>0
$$

such that for energies $E \leq E_{1}, x \in \mathbb{R}^{d}$, and scales $L \in 2 \mathbb{N}$,

$$
\mathbb{P}\left\{\sigma\left(H_{\omega, \Lambda_{L}(x)}^{(1)}\right) \cap(-\infty, E] \neq \emptyset\right\} \leq \mathrm{e}^{-E^{-\frac{d}{2+\varepsilon}}} L^{d},
$$

and hence

$$
\mathbb{P}\left\{H_{\omega, \Lambda_{L}(x)}^{(1)} \geq \min \left\{\left(d \log L-\log p_{n}\right)^{-\frac{2+\varepsilon}{d}}, E_{1}\right\}\right\} \geq 1-p_{n} .
$$

Proceeding as in [18, Proof of Proposition 4.3], for each $x \in \mathbb{R}^{d}$ and scales $L \geq 1$, we consider the event

$$
\Omega_{L, x}=\left\{H_{\omega, \Lambda_{L}(x)}^{(1)} \geq 2 E_{L}^{\prime}\right\},
$$

where

$$
E_{L}^{\prime}=\frac{1}{2}\left(d \log \left(L+\delta_{+}+2\right)-\log p_{n}\right)^{-\frac{2+\varepsilon}{d}},
$$

and conclude that for scales $L \geq \mathcal{L}_{n}^{\prime}=\mathcal{L}_{n}^{\prime}\left(d, u_{-}, \delta_{-}, \mu, p_{0}, \varepsilon\right)$ we have

$$
\mathbb{P}\left\{\Omega_{L, x}\right\} \geq 1-p_{n} \quad \text { for all } x \in \mathbb{R}^{d} .
$$

Now let $\boldsymbol{x} \in \mathbb{R}^{n d}$, and consider the $n$-particle box $\boldsymbol{\Lambda}_{L}^{(n)}(\boldsymbol{x})$. Given $L \geq 1$, we set

$$
\Omega_{L, x}=\bigcap_{i=1}^{n} \Omega_{L, x_{i}},
$$

so

$$
\mathbb{P}\left\{\Omega_{L, \boldsymbol{x}}\right\} \geq 1-n p_{n}=1-p_{0} \quad \text { for } L \geq \mathcal{L}_{n}^{\prime} .
$$

In view of (1.11),

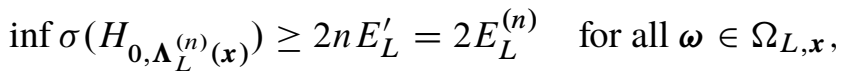

which implies, using $U \geq 0$,

$$
\inf \sigma\left(H_{\Lambda_{L}^{(n)}(x)}\right) \geq 2 E_{L}^{(n)} \text { for all } \omega \in \Omega_{L, x} .
$$

We now fix $\boldsymbol{\omega} \in \Omega_{L, \boldsymbol{x}}$ and let $E \leq E_{L}^{(n)}$ and $\boldsymbol{a}, \boldsymbol{b} \in \boldsymbol{\Lambda}=\boldsymbol{\Lambda}_{L}^{(n)}(\boldsymbol{x})$ with $\|\boldsymbol{a}-\boldsymbol{b}\| \geq \frac{L}{100}$. It follows from (4.3) and Lemma 3.3 that $E \notin \sigma\left(H_{\boldsymbol{\Lambda}_{L}^{(n)}(\boldsymbol{x})}\right)$ and (provided $L$ is sufficiently large)

$$
\left\|\chi_{\boldsymbol{a}} R_{\boldsymbol{\Lambda}}(E) \chi_{\boldsymbol{b}}\right\| \leq \frac{4}{3}\left(E_{L}^{(n)}\right)^{-1} e^{-\frac{L}{201} \sqrt{E_{L}^{(n)}}} .
$$

Thus, given $\theta>0$, there exists $\mathcal{L}_{n}=\mathcal{L}_{n}\left(d, u_{-}, \delta_{ \pm}, \mu, \theta, p_{0}, \varepsilon\right)$, such that for all $L \geq \mathcal{L}_{n}$ and $\boldsymbol{x} \in \mathbb{R}^{n d}$ we have (4.1). 
Remark 4.2. The hypotheses of Theorem 1.6 can be verified in a fixed interval at the bottom of the spectrum at high disorder. To see that, consider

$$
H_{\omega, \lambda}^{(n)}=-\Delta^{(n)}+\lambda V_{\omega}^{(n)}+U,
$$

where $V_{\omega}^{(n)}$ and $U$ are as in Definition 1.1 and $\lambda>0$ is the disorder parameter. $H_{\omega, \lambda}^{(n)}$ can be rewritten as $n$-particle Anderson Hamiltonian in the form of Definition 1.1 by replacing the probability distribution $\mu$ by the probability distribution $\mu_{\lambda}$, where $\mu_{\lambda}$ is the probability distribution of the random variable $\lambda \omega_{0}$, that is, $\mu_{\lambda}(B)=\mu\left(\lambda^{-1} B\right)$ for all Borel sets $B \subset \mathbb{R}$. In particular, $\mu_{\lambda}$ has density $\rho_{\lambda}\left(\omega_{0}\right)=\lambda^{-1} \rho\left(\lambda^{-1} \omega_{0}\right)$.

For simplicity we assume the covering condition

$$
U_{-} \chi_{\Lambda} \leq \sum_{k \in \mathbb{Z}^{d} \cap \Lambda} u(x-k)
$$

for all one-particle boxes $\Lambda$, where $U_{-}>0$. (The condition (4.5) can be guaranteed by requiring $\delta_{-} \geq 2$. If we restrict ourselves to boxes $\Lambda_{L}(x)$ with $x \in \mathbb{Z}^{d}$ and $L$ an odd natural number it suffices to require $\delta_{-} \geq 1$.) In this case it is well known how to proceed in the one-particle case (see [10, 15]): Given $E_{1}>0$, it follows from (4.5) that

$$
\mathbb{P}\left\{H_{\omega, \lambda, \Lambda_{L}(x)}^{(1)} \geq 2 E_{1}\right\} \geq 1-L^{d} \mu_{\lambda}\left\{\left[0,2 E_{1} U_{-}^{-1}\right]\right\} \geq 1-2 E_{1} U_{-}^{-1} \lambda^{-1}\|\rho\|_{\infty} L^{d} .
$$

Proceeding as in the proof of Theorem 4.1, we obtain

$$
\mathbb{P}\left\{H_{\omega, \lambda, \Lambda_{L}^{(n)}(x)}^{(n)} \geq 2 n E_{1}\right\} \geq 1-2 n E_{1} U_{-}^{-1} \lambda^{-1}\|\rho\|_{\infty} L^{d} .
$$

Given $0<p(n)<1$ and $E>0$, we set

$$
\lambda(E, L, p(n))=\frac{2 E\|\rho\|_{\infty} L^{d}}{p(n) U_{-}},
$$

obtaining for all $E>0$

$$
\mathbb{P}\left\{H_{\boldsymbol{\omega}, \lambda, \boldsymbol{\Lambda}_{L}^{(n)}(\boldsymbol{x})}^{(n)} \geq 2 E\right\} \geq 1-p(n) \quad \text { for all } \lambda \geq \lambda(E, L, p(n)) .
$$

To use Lemma 3.3 as in (4.4), we require

$$
\frac{4}{3} E^{-1} e^{-\frac{L}{201} \sqrt{E}} \leq L^{-\theta}
$$

i.e., $L \geq L(E, \theta)$. 
We conclude that, given $0<p(n), E_{0}>0$, and $\theta>0$, for all $L \geq L\left(E_{0}, \theta\right)$ and $\lambda \geq \lambda\left(E_{0}, L, p(n)\right)$ we have

$\mathbb{P}\left\{\Lambda_{L}^{(n)}(\boldsymbol{x})\right.$ is $(\theta, E)$-suitable for $\left.H_{\omega, \lambda}^{(n)}\right\} \geq 1-p(n)$ for all $E \leq E_{0}$.

If we do not assume the covering condition (4.5), we can still prove a large disorder result using [18, Proposition 4.5] for the one-particle case.

\section{The multi-particle bootstrap multiscale analysis}

Theorem 1.6 is proven by induction on $N$, the number of particles. For $N=1$ the theorem was proved by Germinet and Klein [13]. Given $N \geq 2$, we assume the induction hypothesis: Theorem 1.6 holds for $n=1,2, \ldots, N-1$ particles, and prove the theorem for $N$ particles. As in [13], the proof will be done by a bootstrapping argument, making successive use of four multiscale analyses.

Induction hypothesis. Let $N \in \mathbb{N}, N \geq 2$, and $E^{(N)}>0$. For every $\tau \in$ $(0,1)$ there is a length scale $L_{\tau}, \delta_{\tau}>0$, and $0<m_{\tau}^{*} \leq \frac{1}{6} \sqrt{E^{(N)}}$, such that for $n=1,2, \ldots, N-1$ the following holds for all $E \leq E^{(n)}:=2^{N-n} E^{(N)}$.

(i) For all $L \geq L_{\tau}$ and $\boldsymbol{a} \in \mathbb{R}^{n d}$,

$$
\mathbb{P}\left\{\boldsymbol{\Lambda}_{L}^{(n)}(\boldsymbol{a}) \text { is }\left(m_{\tau}^{*}, E\right) \text {-nonregular }\right\} \leq e^{-L^{\tau}} .
$$

(ii) Fix $E<E^{(n)}$ and let $I(E)=\left[E-\delta_{\tau}, E+\delta_{\tau}\right] \cap\left(-\infty, E^{(n)}\right]$. For all $L \geq L_{\tau}$ and all pairs of $n$-particle boxes $\boldsymbol{\Lambda}_{L}^{(n)}(\boldsymbol{a})$ and $\boldsymbol{\Lambda}_{L}^{(n)}(\boldsymbol{b})$ with $d_{H}(\boldsymbol{a}, \boldsymbol{b}) \geq \bar{L}$, we get

$\mathbb{P}\left\{\right.$ there exists $E^{\prime} \in I(E)$ such that

$$
\text { both } \left.\Lambda_{L}^{(n)}(\boldsymbol{a}) \text { and } \boldsymbol{\Lambda}_{L}^{(n)}(\boldsymbol{b}) \text { are }\left(m_{\tau}^{*}, E^{\prime}\right) \text {-nonregular }\right\} \leq e^{-L^{\tau}} .
$$

Lemma 3.9 (ii) will play an important role in the proof of Theorem 1.6. To satisfy its hypotheses, the induction hypothesis specifies $m_{\tau}^{*} \leq \frac{1}{6} \sqrt{E^{(N)}}$ for every $\tau \in(0,1)$, without loss of generality, and sets

$$
E^{(n)}:=2^{N-n} E^{(N)} \text {. }
$$

In this SECTION WE FIX $N \in \mathbb{N}, N \geq 2$, AND AN ENERgy $E^{(N)}>0$, AND ASSUME THAT THE INDUCTION HYPOTHESIS HOLDS FOR THIS $N$ AND $E^{(N)}$.

For partially interactive $N$-particle boxes we immediately get probability estimates from the induction hypothesis. 
Lemma 5.1. Let

$$
\boldsymbol{\Lambda}_{\ell}^{(N)}(\boldsymbol{u})=\boldsymbol{\Lambda}_{\ell}\left(\mathbf{u}_{\mathfrak{f}}\right) \times \boldsymbol{\Lambda}_{\ell}\left(\mathbf{u}_{\mathfrak{g} c}\right)
$$

be a PI $N$-particle box and $\tau \in(0,1)$. Then for $\ell$ large and all $E \leq E^{(N)}$,

$$
\mathbb{P}\left\{\boldsymbol{\Lambda}_{\ell}^{(N)}(\boldsymbol{u}) \text { is }\left(m_{\tau}^{*}(\ell), E\right) \text {-nonregular }\right\} \leq \ell^{N d+1} e^{-\ell^{\tau}},
$$

with

$$
m_{\tau}^{*}(\ell)=m_{\tau}^{*}-\frac{100(n d+1) \log (2 \ell)}{\ell}
$$

$$
\mathbb{P}\left\{\boldsymbol{\Lambda}_{\ell}^{(N)}(\boldsymbol{u}) \text { is }(\theta, E) \text {-nonsuitable }\right\} \leq \ell^{N d+1} e^{-\ell^{\tau}},
$$

for

$$
\theta<\frac{\ell}{\log \ell} \frac{m_{\tau}^{*}}{100}
$$

and

$$
\mathbb{P}\left\{\boldsymbol{\Lambda}_{\ell}^{(N)}(\boldsymbol{u}) \text { is }(\tau, E)-n o n S E S\right\} \leq \ell^{N d+1} e^{-\ell^{\tau}} .
$$

Proof. Let $E \leq E^{(N)}$. It follows from Lemma 3.9 (ii) and the induction hypothesis, using also Lemma 3.2, that for large $\ell$,

$$
\begin{aligned}
& \mathbb{P}\left\{\boldsymbol{\Lambda}_{\ell}^{(N)}(\boldsymbol{u}) \text { is }\left(m_{\tau}^{*}(\ell), E\right) \text {-nonregular }\right\} \\
& \leq \sum_{\mu \in \sigma_{\mathfrak{\jmath} c} \cap\left(-\infty, 2 E^{(N)}\right]} \mathbb{P}\left\{\boldsymbol{\Lambda}_{\ell}\left(\mathbf{u}_{\mathcal{\jmath}}\right) \text { is }\left(m_{\tau}^{*}, E-\mu\right) \text {-nonregular }\right\} \\
& \quad+\sum_{\lambda \in \sigma_{\mathcal{J}} \cap\left(-\infty, 2 E^{(N)}\right]} \mathbb{P}\left\{\boldsymbol{\Lambda}_{\ell}\left(\mathbf{u}_{\mathfrak{\jmath}}\right) \text { is }\left(m_{\tau}^{*}, E-\lambda\right) \text {-nonregular }\right\} \\
& \leq C_{N, d}\left(E^{(N)}\right)^{\frac{N d}{2}} \ell^{N d} e^{-\ell^{\tau}} \\
& \leq \ell^{N d+1} e^{-\ell^{\tau}} .
\end{aligned}
$$

The other estimates now follow from Remark 1.5.

In what follows, we fix $\zeta, \tau, \beta, \zeta_{0}, \zeta_{1}, \zeta_{2}, \gamma$ such that

$$
0<\zeta<\tau<1, \quad \zeta \gamma^{2}<\zeta_{2}
$$

and

$$
0<\zeta<\zeta_{2}<\gamma \zeta_{2}<\zeta_{1}<\gamma \zeta_{1}<\beta<\zeta_{0}<r<\tau<1 \quad \text { with } \zeta \gamma^{2}<\zeta_{2} .
$$


We set

$$
m^{*}=m_{\tau}^{*},
$$

where $m_{\tau}^{*} \leq \frac{1}{6} \sqrt{E^{(N)}}$ is given in the induction hypothesis.

We will use the Wegner estimates of Theorem 2.2 and Corollary 2.3 for $n=$ $1,2, \ldots, N$ particles, which apply to an interval $I \subseteq\left[0, E_{+}\right)$with $|I| \leq 2 \gamma_{n, E_{+}}$. In the multiscale analysis we will need

$$
E_{+}=E^{(n)}:=2^{N-n} E^{(N)}
$$

for the $n$-particles Wegner estimates. For convenience, we take

$$
E_{+}=E^{(1)}=2^{N-1} E^{(N)} \geq E^{(n)} \text { for } n=1,2, \ldots, N .
$$

Note that the constants in these Wegner estimates (including $\gamma_{n, E_{+}}$) are increasing in $n$ and on $E_{+}$, so we will always take the constants for $n=N$ and $E_{+}=E^{(1)}$ (e.g., $\gamma_{N, E_{+}}$). To ensure that the condition $|I| \leq 2 \gamma_{N, E_{1}}$ is always satisfied, we will always take sufficiently large scales $L$, i.e.,

$$
L \geq L\left(\gamma_{N, E_{1}}\right)
$$

such that

$$
L^{-s} \leq \gamma_{N, E_{1}} \quad \text { and } \quad e^{-L^{\beta}} \leq \gamma_{N, E_{1}} .
$$

Moreover, in the following lemmas the conclusions are always assumed to hold for $L$ sufficiently large.

The proof of the induction step proceeds as in [13, 23], with four multi-scale analyses, as in [27], using the toolkit for the multiscale analysis in the continuum given in Section 3. We state all the steps, but refer to [27] for the proofs when they are similar.

\subsection{The first multiscale analysis}

Proposition 5.2. Let $\theta>8 N d$ and $E \leq E^{(N)}$. Take

$$
0<p<p+N d<s<s+2 N d-2<\theta
$$

$Y \geq 4000 N^{N+1}$, and $p_{0}=p_{0}(N)<\frac{1}{2}(2 Y)^{-N d}$. Then there exists a length scale $Z_{0}^{*}$ such that if, for some $L_{0} \geq Z_{0}^{*}$, we have

$$
\sup _{\boldsymbol{x} \in \mathbb{R}^{N d}} \mathbb{P}\left\{\boldsymbol{\Lambda}_{L_{0}}^{(N)}(\boldsymbol{x}) \text { is }(\theta, E) \text {-nonsuitable }\right\} \leq p_{0},
$$

then, setting

$$
L_{k+1}=Y L_{k}, \quad \text { for } k=0,1,2, \ldots,
$$


there exists $K_{0} \in \mathbb{N}$ such that, for every $k \geq K_{0}$,

$$
\sup _{\boldsymbol{x} \in \mathbb{R}^{N d}} \mathbb{P}\left\{\boldsymbol{\Lambda}_{L_{k}}^{(N)}(\boldsymbol{x}) i s(\theta, E) \text {-nonsuitable }\right\} \leq L_{k}^{-p} .
$$

The proof of the proposition uses the following deterministic lemma.

Lemma 5.3. Let $\theta>8 N d$ and $E \leq E^{(N)}$. Take $N d<s<s+2 N d<\theta$. Let $J \in \mathbb{N}, Y \geq 4000 J N^{N+1}, L=Y \ell$, and $\boldsymbol{x} \in \mathbb{R}^{N d}$. Suppose we have the following:

(i) $\boldsymbol{\Lambda}_{L}^{(N)}(\boldsymbol{x})$ is E-suitably nonresonant;

(ii) there are at most $J$ pairwise $\ell$-distant, $(E, \theta)$-nonsuitable boxes in the $\ell$ suitable cover;

(iii) everybox $\boldsymbol{\Lambda}_{t}^{(N)}(\boldsymbol{u}) \subseteq \boldsymbol{\Lambda}_{L}^{(N)}(\boldsymbol{x})$ with $t \in\left\{\left(2 k_{j} \alpha+1\right) \ell ; j=1, \ldots, J N^{N}\right\}$ and $\boldsymbol{u} \in \boldsymbol{x}+\alpha \ell \mathbb{Z}^{N d}$, where $k_{j}$ is given in Remark 3.6, is $E$-suitably nonresonant.

Then the $N$-particle box $\boldsymbol{\Lambda}_{L}^{(N)}(\boldsymbol{x})$ is $(E, \theta)$-suitable for $L$ sufficiently large.

Lemma 5.3 has the same proof as [27, Lemma 3.3]. Prop 5.2 is proved using Lemma 5.3 as [27, Proposition 3.2] is proved using [27, Lemma 3.3].

\subsection{The second multiscale analysis}

Proposition 5.4. Let $E \leq E^{(N)}, p>0, \theta>0,1<\gamma<1+\frac{p}{p+2 N d}$. Then there exists a length scale $Z_{1}^{*}$ such that if for some $L_{0} \geq Z_{1}^{*}$ we can verify

$$
\sup _{\boldsymbol{x} \in \mathbb{R}^{N d}} \mathbb{P}\left\{\boldsymbol{\Lambda}_{L_{0}}^{(N)}(\boldsymbol{x}) \text { is }\left(m_{0}, E\right) \text {-nonregular }\right\} \leq L_{0}^{-p},
$$

where $\theta \frac{\log L_{0}}{L_{0}} \leq m_{0}<m^{*}$, then, setting

$$
L_{k+1}=L_{k}^{\gamma}, \quad \text { for } k=1,2, \ldots,
$$

we get

$$
\sup _{\boldsymbol{x} \in \mathbb{R}^{N d}} \mathbb{P}\left\{\boldsymbol{\Lambda}_{L_{k}}^{(N)}(\boldsymbol{x}) \text { is }\left(\frac{m_{0}}{2}, E\right) \text {-nonregular }\right\} \leq L_{k}^{-p} \quad \text { for all } k=0,1,2, \ldots
$$

To prove the proposition we use the following deterministic lemma. 
Lemma 5.5. Let $E \leq E^{(N)}, L=\ell^{\gamma}, J \in \mathbb{N}, m_{0}>0$, and

$$
m_{\ell} \in\left[\frac{1}{\ell^{\kappa}}, m_{0}\right], \quad \text { where } 0<\kappa<\min \{\gamma-1, \gamma(1-\beta), 1\} \text {. }
$$

Suppose that we have the following:

(i) $\boldsymbol{\Lambda}_{L}^{(N)}(\boldsymbol{x})$ is E-nonresonant;

(ii) there are at most $J$ pairwise $\ell$-distant, $\left(E, m_{\ell}\right)$-nonregular boxes in the suitable cover;

(iii) every box $\boldsymbol{\Lambda}_{t}^{(N)}(\boldsymbol{u}) \subseteq \boldsymbol{\Lambda}_{L}^{(N)}(\boldsymbol{x})$ with $t \in\left\{\left(2 k_{j} \alpha+1\right) \ell ; j=1, \cdots, J N^{N}\right\}$ and $\boldsymbol{u} \in \boldsymbol{x}+\alpha \ell \mathbb{Z}^{N d}$, where $k_{j}$ is given in Remark 3.6, is E-nonresonant.

Then $\boldsymbol{\Lambda}_{L}^{(N)}(\boldsymbol{x})$ is $\left(E, m_{L}\right)$-regular for L large, where

$$
m_{\ell} \geq m_{L} \geq m_{\ell}-\frac{1}{2 \ell^{\kappa}} \geq \frac{1}{L^{\kappa}} .
$$

Lemma 5.5 and Proposition 5.4 are proved in the same way as [27, Lemma 3.5 and Proposition 3.4].

\subsection{The third multiscale analysis}

Proposition 5.6. Let $E \leq E^{(N)}, 0<\zeta_{1}<\zeta_{0}<1$ as in (5.3), and assume $Y \geq\left(3800 N^{N+1}\right)^{\frac{1}{1-\xi_{0}}}$. Then there exists $Z_{2}^{*}>L_{\tau}$ such that, if for some scale $L_{0}>Z_{2}^{*}$ we have

$$
\sup _{\boldsymbol{x} \in \mathbb{R}^{N d}} \mathbb{P}\left\{\boldsymbol{\Lambda}_{L_{0}}^{(N)}(\boldsymbol{x}) \text { is }\left(\zeta_{0}, E\right)-n o n S E S\right\} \leq\left(2(2 Y)^{N d}\right)^{-\frac{1}{Y^{\zeta_{0}-1}}},
$$

then, setting

$$
L_{k+1}=Y L_{k}, \quad k=0,1,2, \ldots,
$$

there exists $K_{1} \in \mathbb{N}$ such that, for every $k \geq K_{1}$,

$$
\sup _{\boldsymbol{x} \in \mathbb{R}^{N d}} \mathbb{P}\left\{\boldsymbol{\Lambda}_{L_{k}}(\boldsymbol{x}) \text { is }\left(\zeta_{0}, E\right)-n o n S E S\right\} \leq e^{-L_{k}^{\zeta_{1}}}
$$

As a consequence, for every $k \geq K_{1}$, we have

$$
\sup _{\boldsymbol{x} \in \mathbb{R}^{N d}} \mathbb{P}\left\{\boldsymbol{\Lambda}_{L_{k}}(\boldsymbol{x}) \text { is }\left(L_{k}^{\zeta_{0}-1}, E\right) \text {-nonregular }\right\} \leq e^{-L_{k}^{\zeta_{1}}} .
$$

The proof of proposition uses the following deterministic lemma. 
Lemma 5.7. Let $E \leq E^{(N)}, L=Y \ell$, where $Y \geq\left(3800 N^{N+1}\right)^{\frac{1}{1-\zeta_{0}}}$, and set

$$
J=\left\lfloor Y^{\zeta_{0}}\right\rfloor,
$$

the largest integer $\leq Y^{\zeta_{0}}$. Suppose the following are true:

(i) $\boldsymbol{\Lambda}_{L}^{(N)}(\boldsymbol{x})$ is E-nonresonant;

(ii) there are at most $J$ pairwise $\ell$-distant, $\left(E, \zeta_{0}\right)$-nonSES boxes in the suitable cover;

(iii) everybox $\boldsymbol{\Lambda}_{t}^{(N)}(\boldsymbol{u}) \subseteq \boldsymbol{\Lambda}_{L}^{(N)}(\boldsymbol{x})$ with $t \in\left\{\left(2 k_{j} \alpha+1\right) \ell ; j=1, \cdots, J N^{N}\right\}$ and $\boldsymbol{u} \in \boldsymbol{x}+\alpha \ell \mathbb{Z}^{N d}$, where $k_{j}$ is given in Remark 3.6, is E-nonresonant.

Then $\boldsymbol{\Lambda}_{L}^{(N)}(\boldsymbol{x})$ is $\left(E, \zeta_{0}\right)$-SES, provided $\ell$ is sufficiently large.

Lemma 5.7 and Proposition 5.6 are proved in the same way as [27, Lemma 3.7 and Proposition 3.6].

5.4. The fourth multiscale analysis. We fix $\zeta, \tau, \beta, \zeta_{1}, \zeta_{2}, \gamma$ as in (5.3).

\subsubsection{The single energy multiscale analysis}

Proposition 5.8. There exists a length scale $Z_{3}^{*}$ such that, given an energy $E \leq$ $E^{(N)}$, if for some $L_{0} \geq Z_{3}^{*}$ we can verify

$$
\sup _{\boldsymbol{a} \in \mathbb{R}^{N d}} \mathbb{P}\left\{\boldsymbol{\Lambda}_{L_{0}}^{(N)}(\boldsymbol{a}) \text { is }\left(m_{0}, E\right) \text {-nonregular }\right\} \leq e^{-L_{0}^{\zeta_{1}}},
$$

where $L_{0}^{\zeta_{0}-1} \leq m_{0}<m^{*}$, then, for sufficiently large $L$,

$$
\sup _{\boldsymbol{a} \in \mathbb{R}^{N d}} \mathbb{P}\left\{\boldsymbol{\Lambda}_{L}^{(N)}(\boldsymbol{a}) \text { is }\left(\frac{m_{0}}{2}, E\right) \text {-nonregular }\right\} \leq e^{-L^{\zeta_{2}}} .
$$

Proposition 5.8 is proved first for a sequence of length scale $L_{k}$ similarly to Proposition 5.4; to obtain the sub-exponential decay of probabilities we choose $J$, the number of bad boxes, dependent on the scale $L$ as in the proof of Proposition 5.19 below. To obtain Proposition 5.8 as stated, that is, for all sufficiently large scales, we prove a slightly more general result.

Definition 5.9. Let $E \in \mathbb{R}$. An $N$-particle box, $\boldsymbol{\Lambda}_{L}^{(N)}(\boldsymbol{x})$, is $\left(E, m_{L}\right)$-good when it is $\left(E, m_{L}\right)$-regular and $E$-nonresonant.

Lemma 5.10. Let $\boldsymbol{\Lambda}_{L}^{(N)}(\boldsymbol{x})$ be an $N$-particle box, $\gamma>1, \ell=L^{\frac{1}{\gamma^{\prime}}}$ with $\gamma \leq \gamma^{\prime} \leq$ $\gamma^{2}$, and $m>0$. Let $E \leq E^{(N)}$, and suppose every box in the suitable cover is $(E, m)$-good. Then $\boldsymbol{\Lambda}_{L}^{(N)}(\boldsymbol{x})$ is $\left(E, \frac{m}{2}\right)$-good for large $L$. 
This lemma is just [18, Lemma 3.16].

Lemma 5.11. Let $E_{1} \leq E^{(N)}, \zeta_{2} \in(\zeta, \tau)$, and $\gamma \in\left(1, \frac{1}{\zeta_{2}}\right)$ with $\zeta \gamma^{2}<\zeta_{2}$. Assume there exists a mass $m_{\zeta_{2}}>0$ and a length scale

$$
L_{0}=L_{0}\left(\zeta_{2}\right)
$$

such that, taking

$$
L_{k+1}=L_{k}^{\gamma} \text { for } k=0,1, \ldots
$$

we have

$$
\sup _{\boldsymbol{a} \in \mathbb{R}^{N d}} P\left\{\boldsymbol{\Lambda}_{L_{k}}^{(N)}(\boldsymbol{a}) \text { is not }\left(m_{\zeta_{2}}, E_{1}\right) \text {-good }\right\} \leq e^{-L_{k}^{\zeta_{2}}} \quad \text { for } k=0,1, \ldots
$$

Then there exists $L_{\zeta}$ such that, for every $L \geq L_{\zeta}$,

$$
\sup _{\boldsymbol{a} \in \mathbb{R}^{N d}} P\left\{\boldsymbol{\Lambda}_{L}^{(N)}(\boldsymbol{a}) \text { is not }\left(m_{\zeta_{2}}, E_{1}\right)-\operatorname{good}\right\} \leq e^{-L^{\zeta}} .
$$

The proof of Lemma 5.11 is straightforward (see [27, Lemma 3.11]).

\subsubsection{The energy interval multiscale analysis}

Lemma 5.12. Let $\boldsymbol{\Lambda}_{L}^{(N)}(\boldsymbol{x})$ be an $N$-particle box and $m>0$. Let $E_{0} \leq E^{(N)}$, and suppose that

(i) $\boldsymbol{\Lambda}_{L}^{(N)}(\boldsymbol{x})$ is $\left(m, E_{0}\right)$-regular,

(ii) $\operatorname{dist}\left(\sigma\left(H_{\boldsymbol{\Lambda}_{L}^{(N)}(\boldsymbol{x})}\right), E_{0}\right) \geq e^{-L^{\beta}}$, i.e., $\left\|R_{\boldsymbol{\Lambda}_{L}^{(N)}(\boldsymbol{x})}\left(E_{0}\right)\right\| \leq e^{L^{\beta}}$.

Then $\Lambda_{L}^{(N)}(\boldsymbol{x})$ is $\left(m-\frac{100 \log 2}{L}, E\right)$-good for every $E \in I=\left(E_{0}-\eta, E_{0}+\eta\right)$, where $\eta=\frac{1}{2} e^{-m L-2 L^{\beta}}$.

Lemma 5.12 is proved as [27, Lemma 3.12].

Proposition 5.6, combined with Theorem 2.2 and Lemma 5.12, yields the following proposition.

Proposition 5.13. Let $0<\zeta_{2}<\zeta_{1}<\zeta_{0}<1$, and assume the conclusions of Proposition 5.6. There exists scales $L_{k}, k=1,2, \ldots$, such that

$$
\lim _{k \rightarrow \infty} L_{k}=\infty
$$

with the following property. Let

$$
m_{k}=\left(L_{k}^{\zeta_{0}-1}-\frac{100 \log 2}{L_{k}}\right) \quad \text { and } \quad \eta_{k}=\frac{1}{2} e^{-L_{k}^{\zeta_{0}}-2 L_{k}^{\beta}}
$$


Then, for all $E_{0} \leq E^{(N)}$,

$$
\begin{gathered}
\sup _{\boldsymbol{x} \in \mathbb{R}^{N d}} \mathbb{P}\left\{\text { there exists } E \in\left(E_{0}-\eta_{k}, E_{0}+\eta_{k}\right)\right. \text { such that } \\
\left.\boldsymbol{\Lambda}_{L_{k}}(\boldsymbol{x}) \text { is }\left(m_{k}, E\right) \text {-nonregular }\right\} \leq e^{-L_{k}^{\zeta_{1}},}
\end{gathered}
$$

and

$$
\begin{gathered}
\sup _{\boldsymbol{x} \in \mathbb{R}^{N d}} \mathbb{P}\left\{\text { there exists } E \in\left(E_{0}-\eta_{k}, E_{0}+\eta_{k}\right)\right. \text { such that } \\
\left.\boldsymbol{\Lambda}_{L_{k}}(\boldsymbol{x}) \text { is not }\left(m_{k}, E\right) \text {-good }\right\} \leq e^{-L_{k}^{\xi_{2}}} .
\end{gathered}
$$

We now take $L=\ell^{\gamma}$.

Definition 5.14. Let

$$
\Lambda_{L}^{(N)}(\boldsymbol{x})=\Lambda_{L}\left(\mathbf{x}_{\mathfrak{f}}\right) \times \Lambda_{L}\left(\mathbf{x}_{\mathfrak{\jmath} c}\right)
$$

be a PI $N$-particle box with the usual $\ell$ suitable cover, and consider an energy $E \in \mathbb{R}$.

(i) $\boldsymbol{\Lambda}_{L}^{(N)}(\boldsymbol{x})$ is not E-Lregular (for "left regular") when there are two boxes in the suitable cover of $\boldsymbol{\Lambda}_{L}\left(\mathbf{x}_{\mathfrak{f}}\right)$ that are $\ell$-distant and $\left(m^{*}, E-\mu\right)$-nonregular for some $\mu \in \sigma\left(H_{\boldsymbol{\Lambda}_{L}\left(\mathbf{x}_{\mathfrak{j}} c\right)}\right) \cap\left(-\infty, 2 E^{(N)}\right]$.

(ii) $\boldsymbol{\Lambda}_{L}^{(N)}(\boldsymbol{x})$ is not $E$-Rregular (for "right regular") when there are two boxes in the suitable cover of $\boldsymbol{\Lambda}_{L}\left(\mathbf{x}_{\mathfrak{j} c}\right)$ that are $\ell$-distant and $\left(m^{*}, E-\lambda\right)$-nonregular for some $\lambda \in \sigma\left(H_{\boldsymbol{\Lambda}_{L}\left(\mathbf{x}_{\jmath}\right)}\right) \cap\left(-\infty, 2 E^{(N)}\right]$.

(iii) $\boldsymbol{\Lambda}_{L}^{(N)}(\boldsymbol{x})$ is $E$-preregular when $\boldsymbol{\Lambda}_{L}^{(N)}(\boldsymbol{x})$ is $E$-Lregular and $E$-Rregular.

Lemma 5.15. Let $E_{0} \leq E^{(N)}$ such that

$$
I=\left[E_{0}-\delta_{\tau}, E_{0}+\delta_{\tau}\right] \subseteq\left(-\infty, 2 E^{(N)}\right],
$$

and consider a PI N-particle box

$$
\boldsymbol{\Lambda}_{L}^{(N)}(\boldsymbol{x})=\boldsymbol{\Lambda}_{L}\left(\mathbf{x}_{\mathfrak{f}}\right) \times \boldsymbol{\Lambda}_{L}\left(\mathbf{x}_{\mathfrak{f} c}\right) .
$$

Then

$$
\mathbb{P}\left\{\boldsymbol{\Lambda}_{L}^{(N)}(\boldsymbol{x}) \text { is not E-Lregular for some } E \in I\right\} \leq L^{3 N d} e^{-\ell^{\tau}},
$$

and

(ii) $\quad \mathbb{P}\left\{\boldsymbol{\Lambda}_{L}^{(N)}(\boldsymbol{x})\right.$ is not E-Lregular for some $\left.E \in I\right\} \leq L^{3 N d} e^{-\ell^{\tau}}$.

We conclude that for $L$ sufficiently large,

(iii) $\mathbb{P}\left\{\boldsymbol{\Lambda}_{L}^{(N)}(\boldsymbol{x})\right.$ is not E-preregular for some $\left.E \in I\right\} \leq 2 L^{3 N d} e^{-\ell^{\tau}}$. 
Lemma 5.15 has the same proof as [27, Lemma 3.15].

Definition 5.16. Let

$$
\Lambda_{L}^{(N)}(\boldsymbol{x})=\Lambda_{L}\left(\mathbf{x}_{\mathcal{J}}\right) \times \Lambda_{L}\left(\mathbf{x}_{\mathfrak{J} c}\right)
$$

be a PI $N$-particle box, and consider an energy $E \leq E^{(N)}$.

(i) $\boldsymbol{\Lambda}_{L}^{(N)}(\boldsymbol{x})$ is E-left nonresonant (or LNR) when for every box $\boldsymbol{\Lambda}_{\left(2 k_{j}+1\right) \ell}(\boldsymbol{a})$ with $\boldsymbol{\Lambda}_{\left(2 k_{j}+1\right) \ell}(\boldsymbol{a}) \subseteq \boldsymbol{\Lambda}_{L}\left(\boldsymbol{x}_{\mathfrak{J}}\right), \boldsymbol{a} \in \boldsymbol{x}_{\mathcal{J}}+\alpha \ell \mathbb{Z}^{|\mathcal{J}| d}$ and $j \in\left\{1,2, \ldots|\mathcal{J}|^{|\mathcal{F}|}\right\}$, is $(E-\mu)$-nonresonant for every $\mu \in \sigma\left(H_{\boldsymbol{\Lambda}_{L}\left(\boldsymbol{u}_{\mathfrak{g}} c\right)}\right) \cap\left(-\infty, 2 E^{(N)}\right]$. Otherwise we say $\boldsymbol{\Lambda}_{L}^{(N)}(\boldsymbol{x})$ is that $E$-left resonant (or LR).

(ii) $\boldsymbol{\Lambda}_{L}^{(N)}(\boldsymbol{x})$ is E-right nonresonant (or RNR) when for every box $\boldsymbol{\Lambda}_{\left(2 k_{j}+1\right) \ell}(\boldsymbol{a}) \subseteq$ $\boldsymbol{\Lambda}_{L}\left(\boldsymbol{x}_{\mathfrak{J}}\right)$ with $\boldsymbol{a} \in \boldsymbol{x}_{\mathfrak{J} c}+\alpha \ell \mathbb{Z}^{\left|\mathcal{J}^{c}\right| d}$ and $j \in\left\{1,2, \ldots\left|\mathcal{J}^{c}\right|^{\mid \mathcal{J}^{c}}\right\}$ is $(E-\lambda)$-nonresonant for every $\lambda \in \sigma\left(H_{\boldsymbol{\Lambda}_{L}\left(\boldsymbol{x}_{\mathfrak{g}}\right)}\right) \cap\left(-\infty, 2 E^{(N)}\right]$. Otherwise we say that $\Lambda_{L}^{(N)}(\boldsymbol{x})$ is E-right resonant (or RR).

(iii) We say $\boldsymbol{\Lambda}_{L}^{(N)}(\boldsymbol{x})$ is E-highly nonresonant (or HNR) when for $\boldsymbol{\Lambda}_{L}^{(N)}(\boldsymbol{x})$ is $E$-nonresonant, $E$-LNR, and $E$-RNR.

Lemma 5.17. Let $E \leq E^{(N)}$ and $\Lambda_{L}^{(N)}(\boldsymbol{x})=\boldsymbol{\Lambda}_{L}\left(\mathbf{x}_{\mathcal{J}}\right) \times \Lambda_{L}\left(\mathbf{x}_{\mathcal{J} c}\right)$ be a PI N-particle box. Assume that

(i) $\boldsymbol{\Lambda}_{L}^{(N)}(\boldsymbol{x})$ is $E-H N R$ and

(ii) $\boldsymbol{\Lambda}_{L}^{(N)}(\boldsymbol{x})$ is E-preregular.

Then $\boldsymbol{\Lambda}_{L}^{(N)}(\boldsymbol{x})$ is $(m(L), E)$-regular for sufficiently large $L$, where

$$
m(L)=m^{*}-\frac{1}{2 L^{\kappa}}-\frac{100(n d+1) \log (2 L)}{L} .
$$

Proof. Applying Lemma 3.9(ii), it is sufficient to prove that there exists $m \leq$ $\frac{1}{6} \sqrt{E^{(N)}}$ such that $\Lambda_{L}\left(\mathbf{x}_{\mathfrak{f}}\right)$ is $(m, E-\mu)$-regular for every $\mu \in \sigma_{\mathfrak{J} c} \cap\left(-\infty, 2 E^{(N)}\right]$ and $\boldsymbol{\Lambda}_{L}\left(\mathbf{x}_{\mathcal{J}} \mathbf{c}\right)$ is $(m, E-\lambda)$-regular for every $\lambda \in \sigma_{\mathfrak{J}} \cap\left(-\infty, 2 E^{(N)}\right]$. Then we can conclude that $\Lambda_{L}^{(N)}(\boldsymbol{x})$ is $\left(m-\frac{100(n d+1) \log (2 L)}{L}, E\right)$-regular.

Let $\mu \in \sigma_{\mathcal{J} c} \cap\left(-\infty, 2 E^{(N)}\right]$. Since $\Lambda_{L}^{(N)}(\boldsymbol{u})$ is $E$-preregular; thus it is $E$-Lregular, which implies there cannot be two boxes in the suitable cover of $\boldsymbol{\Lambda}_{L}\left(\boldsymbol{x}_{\mathfrak{J}}\right)$ that are $\ell$-distant and $\left(m^{*}, E-\mu\right)$-nonregular. Moreover, $\boldsymbol{\Lambda}_{L}^{(N)}(\boldsymbol{u})$ is $E$-HNR; thus it is $E$-LNR, which implies every box $\boldsymbol{\Lambda}_{\left(2 k_{j}+1\right) \ell}(\boldsymbol{a})$ with $\boldsymbol{\Lambda}_{\left(2 k_{j}+1\right) \ell}(\boldsymbol{a}) \subseteq$ $\boldsymbol{\Lambda}_{L}\left(\boldsymbol{x}_{\mathfrak{f}}\right), \boldsymbol{a} \in \boldsymbol{x}_{\mathfrak{J}}+\alpha \ell \mathbb{Z}^{|\mathcal{J}| d}$ and $j \in\left\{1,2, \ldots,|\mathcal{J}|^{|\mathcal{J}|}\right\}$, is $(E-\mu)$-nonresonant. By Lemma 5.5, we have $\boldsymbol{\Lambda}_{L}\left(\mathbf{x}_{\mathfrak{f}}\right)$ is $\left(m^{*}-\frac{1}{2 L^{\kappa}}, E-\mu\right)$-regular. A similar argument will apply for $\lambda \in \sigma_{\mathcal{J}} \cap\left(-\infty, 2 E^{(N)}\right]$. We conclude that $\boldsymbol{\Lambda}_{L}^{(N)}(\boldsymbol{x})$ is $\left(m^{*}-\frac{1}{2 L^{\kappa}}-\frac{100(n d+1) \log (2 L)}{L}, E\right)$-regular. 
Lemma 5.18. Let $E \leq E^{(N)}$, and

$$
\boldsymbol{\Lambda}_{L}^{(N)}(\boldsymbol{x})=\boldsymbol{\Lambda}_{L}\left(\mathbf{x}_{\mathfrak{f}}\right) \times \boldsymbol{\Lambda}_{L}\left(\mathbf{x}_{\mathfrak{j} c}\right)
$$

be a PI N-particle box.

(i) If $\boldsymbol{\Lambda}_{L}^{(N)}(\boldsymbol{x})$ is E-right resonant, then there exists an $N$-particle rectangle

$$
\boldsymbol{\Lambda}=\boldsymbol{\Lambda}_{L}\left(\boldsymbol{x}_{\mathfrak{J}}\right) \times \boldsymbol{\Lambda}_{\left(2 k_{j} \alpha+1\right) \ell}(\boldsymbol{u}),
$$

where $j \in\left\{1,2, \ldots,\left|\mathcal{J}^{c}\right|^{\left|\mathcal{F}^{c}\right|}\right\}, \boldsymbol{u} \in \boldsymbol{x}_{\mathfrak{g} c}+\alpha \ell \mathbb{Z}^{\left|\mathcal{I}^{c}\right| d}$, and $\boldsymbol{\Lambda}_{\left(2 k_{j} \alpha+1\right) \ell}(\boldsymbol{u}) \subseteq$ $\boldsymbol{\Lambda}_{L}\left(\boldsymbol{x}_{\mathfrak{j} c}\right)$, such that

$$
\operatorname{dist}(\sigma(\boldsymbol{\Lambda}), E)<\frac{1}{2} e^{-\left(\left(2 k_{j} \alpha+1\right) \ell\right)^{\beta}} \leq \frac{1}{2} e^{-\ell^{\beta}} .
$$

(ii) If $\boldsymbol{\Lambda}_{L}^{(N)}(\boldsymbol{x})$ is E-left resonant, then there exists an $N$-particle rectangle

$$
\boldsymbol{\Lambda}=\boldsymbol{\Lambda}_{\left(2 k_{j} \alpha+1\right) \ell}(\boldsymbol{u}) \times \boldsymbol{\Lambda}_{L}\left(\boldsymbol{x}_{\mathfrak{J} c}\right),
$$

where $j \in\left\{1,2, \ldots,|\mathcal{f}|^{|\mathcal{J}|}\right\}, \boldsymbol{u} \in \boldsymbol{x}_{\mathfrak{J}}+\alpha \ell \mathbb{Z}^{|\mathcal{J}| d}$, and $\boldsymbol{\Lambda}_{\left(2 k_{j} \alpha+1\right) \ell}(\boldsymbol{u}) \subseteq \boldsymbol{\Lambda}_{L}\left(\boldsymbol{u}_{\mathfrak{J}}\right)$, such that

$$
\operatorname{dist}(\sigma(\boldsymbol{\Lambda}), E)<\frac{1}{2} e^{-\left(\left(2 k_{j} \alpha+1\right) \ell\right)^{\beta}} \leq \frac{1}{2} e^{-\ell^{\beta}} .
$$

Proof. Let $E \leq E^{(N)}$ and

$$
\boldsymbol{\Lambda}_{L}^{(N)}(\boldsymbol{x})=\boldsymbol{\Lambda}_{L}\left(\mathbf{x}_{\mathfrak{f}}\right) \times \boldsymbol{\Lambda}_{L}\left(\mathbf{x}_{\mathfrak{j} c}\right)
$$

be a PI $N$-particle box. Suppose $\Lambda_{L}^{(N)}(\boldsymbol{x})$ is $E$-right resonant. (The same argument applies if $\boldsymbol{\Lambda}_{L}^{(N)}(\boldsymbol{x})$ is $E$-left resonant.) Then we can find $\lambda \in \sigma\left(H_{\boldsymbol{\Lambda}_{L}\left(\boldsymbol{x}_{\mathfrak{f}}\right)}\right) \cap$ $\left(-\infty, 2 E^{(N)}\right]$ and an $(N-|\mathcal{J}|)$-particle box, $\boldsymbol{\Lambda}_{\left(2 k_{j} \alpha+1\right) \ell}(\boldsymbol{u}) \subseteq \boldsymbol{\Lambda}_{L}\left(\boldsymbol{x}_{\mathfrak{g} c}\right)$, with $\boldsymbol{u} \in$ $\boldsymbol{x}_{\mathfrak{J} c}+\alpha \ell \mathbb{Z}^{N d}$ and $j \in\left\{1,2, \ldots,\left|\mathcal{J}^{c}\right|^{\mid \mathcal{F}^{c}} \mid\right.$, such that $\boldsymbol{\Lambda}_{\left(2 k_{j} \alpha+1\right) \ell}(\boldsymbol{u})$ is $(E-\lambda)$-resonant, so there exists $\eta \in \sigma\left(H_{\boldsymbol{\Lambda}_{\left(2 k_{j} \alpha+1\right) \ell}(\boldsymbol{x})}\right)$ such that

$$
|E-\lambda-\eta|<\frac{1}{2} e^{-\left(\left(2 k_{j} \alpha+1\right) \ell\right)^{\beta}} .
$$

Moreover, $\boldsymbol{\Lambda}_{L}\left(\mathbf{x}_{\mathfrak{f}}\right) \times \boldsymbol{\Lambda}_{L}\left(\mathbf{x}_{\mathfrak{f} c}\right)$ is PI and $\boldsymbol{\Lambda}_{\left(2 k_{j} \alpha+1\right) \ell}(\boldsymbol{u}) \subseteq \boldsymbol{\Lambda}_{L}\left(\boldsymbol{x}_{\mathfrak{J} c}\right)$, so if we take $\boldsymbol{\Lambda}=\boldsymbol{\Lambda}_{L}\left(\boldsymbol{x}_{\mathfrak{J}}\right) \times \boldsymbol{\Lambda}_{\left(2 k_{j} \alpha+1\right) \ell}(\boldsymbol{u})$, then we get

$$
\sigma\left(H_{\boldsymbol{\Lambda}}\right)=\sigma\left(H_{\boldsymbol{\Lambda}_{L}\left(\boldsymbol{x}_{\mathfrak{f}}\right)}\right)+\sigma\left(H_{\boldsymbol{\Lambda}_{\left(2 k_{j} \alpha+1\right) \ell}(\boldsymbol{u})}\right) .
$$

Hence, if a PI $N$-particle box $\Lambda_{L}^{(N)}(\boldsymbol{x})=\boldsymbol{\Lambda}_{L}\left(\mathbf{x}_{\mathfrak{f}}\right) \times \boldsymbol{\Lambda}_{L}\left(\mathbf{x}_{\mathfrak{j}}\right)$ is $E$-right resonant, then there exists an $N$-particle box $\boldsymbol{\Lambda}=\boldsymbol{\Lambda}_{L}\left(\boldsymbol{x}_{\mathfrak{\gamma}}\right) \times \boldsymbol{\Lambda}_{\left(2 k_{j} \alpha+1\right) \ell}(\boldsymbol{u})$, where $\boldsymbol{\Lambda}_{\left(2 k_{j} \alpha+1\right) \ell}(\boldsymbol{u}) \subseteq \boldsymbol{\Lambda}_{L}\left(\boldsymbol{x}_{\mathfrak{g}}\right)$, such that

$$
\operatorname{dist}\left(\sigma\left(H_{\boldsymbol{\Lambda}}\right), E\right)<\frac{1}{2} e^{-\left(\left(2 k_{j} \alpha+1\right) \ell\right)^{\beta}} .
$$


We now state the energy interval multiscale analysis. Given $m>0, L \in \mathbb{N}$, $\boldsymbol{x}, \boldsymbol{y} \in \mathbb{Z}^{N d}$, and an interval $I$, we define the event

$$
\begin{aligned}
R(m, I, \boldsymbol{x}, \boldsymbol{y}, L, N)= & \{\text { there exists } E \in I \text { such that } \\
& \text { both } \left.\boldsymbol{\Lambda}_{L}^{(N)}(\boldsymbol{x}) \text { and } \boldsymbol{\Lambda}_{L}^{(N)}(\boldsymbol{y}) \text { are not }(m, E) \text {-regular }\right\} .
\end{aligned}
$$

Proposition 5.19. . Let $\zeta, \tau, \beta, \zeta_{1}, \zeta_{2}, \gamma$ as in (5.3) and $0<m_{0}<m^{*}$. There exists a length scale $Z_{3}^{*}$ such that, given a closed interval $I \subseteq\left(-\infty, E^{(N)}\right]$, if for some $L_{0} \geq Z_{3}^{*}$ we can verify

$$
\mathbb{P}\left\{R\left(m_{0}, I, \boldsymbol{x}, \boldsymbol{y}, L_{0}, N\right)\right\} \leq e^{-L_{0}^{\zeta_{2}}}
$$

for every pair of partially separated $N$-particle boxes $\Lambda_{L_{0}}^{N}(\boldsymbol{x})$ and $\boldsymbol{\Lambda}_{L_{0}}^{(N)}(\boldsymbol{y})$, then, setting

$$
L_{k+1}=L_{k}^{\gamma}=L_{0}^{\gamma^{k}} \quad \text { for } k=0,1,2, \ldots
$$

for every pair of partially separated $N$-particle boxes $\boldsymbol{\Lambda}_{L_{k}}^{(N)}(\boldsymbol{x})$ and $\boldsymbol{\Lambda}_{L_{k}}^{(N)}(\boldsymbol{y})$,

$$
\begin{aligned}
\mathbb{P}\{ & \left.R\left(\frac{m_{0}}{2}, I, \boldsymbol{x}, \boldsymbol{y}, L_{k}, N\right)\right\} \\
\leq & \mathbb{P}\{\text { there exists } E \in I \text { such that } \\
& \left.\quad \boldsymbol{\Lambda}_{L_{k}}^{(N)}(\boldsymbol{x}) \text { and } \boldsymbol{\Lambda}_{L_{k}}^{(N)}(\boldsymbol{y}) \text { are not }\left(\frac{m_{0}}{2}, E\right) \text {-good }\right\} \\
\leq & e^{-L_{k}^{\zeta_{2}}} .
\end{aligned}
$$

Proposition 5.19 is proved in the same way as [27, Propositions 3.19 and 3.21]. The dependence of the length scale $Z_{3}^{*}$ on $E^{(N)}$ (not present in [27]) comes from the use of Theorem 2.2 and Corollary 2.3.

5.5. Completing the proof of the bootstrap multiscale analysis. Proceeding as in [13, Section 6], Theorem 1.6 follows from Propositions 5.2, 5.4, and 5.6, plus Proposition 5.8 for Part (i) (the single energy bootstrap multiscale analysis), and Propositions 5.13 and 5.19 (the energy interval bootstrap multiscale analysis).

\section{From the bootstrap multiscale analysis to localization}

Corollary 1.7 is proved from Theorem 1.6 along the lines of the proofs of the corresponding statements in $[11,13,17,18])$, similarly to the proof of [27, Corollary 1.7] from [27, Theorem 1.5]. 


\section{A. The almost-sure spectrum of the $n$-particle Anderson Hamiltonian}

Proposition A.1. Let $\Sigma^{(n)}$ be the almost-sure spectrum of the n-particle Anderson Hamiltonian $H_{\omega}^{(n)}$ as in Definition 1.1. Then $\Sigma^{(n)}=[0, \infty)$

Proof. Clearly $\Sigma^{(n)} \subset[0, \infty)$. We need to prove $[0, \infty) \subset \Sigma^{(n)}$. So let $\lambda \in[0, \infty)$ and $\varepsilon>0$. Since $\sigma\left(-\Delta^{(n)}\right)=[0, \infty)$, there exists $\psi \in C^{2}\left(\mathbb{R}^{n d}\right)$ with $\|\psi\|=1$ and a box $\boldsymbol{\Lambda}_{L}^{(n)}(\boldsymbol{x})$ such that supp $\psi \subseteq \boldsymbol{\Lambda}_{L}^{(n)}(\boldsymbol{x})$ and $\left\|\left(-\Delta^{(n)}-\lambda\right) \psi\right\| \leq \frac{\epsilon}{2}$. Without loss of generality we can assume that $U=0$ on $\Lambda_{L}^{(n)}(\boldsymbol{x})$, so $H_{\omega}^{(n)} \psi=H_{0, \boldsymbol{\omega}}^{(n)} \psi$. If we have $V_{\boldsymbol{\omega}}^{(n)} \chi_{\boldsymbol{\Lambda}_{L}^{(n)}(\boldsymbol{x})} \leq \frac{\epsilon}{2}$, we conclude that $\left\|\left(H_{\boldsymbol{\omega}}^{(n)}-\lambda\right) \psi\right\| \leq \varepsilon$, and hence $\operatorname{dist}\left(\lambda, \sigma\left(H_{\omega}^{(n)}\right)\right) \leq \varepsilon$. Thus,

$$
\mathbb{P}\left\{\operatorname{dist}\left(\lambda, \sigma\left(H_{\omega}^{(n)}\right)\right) \leq \varepsilon\right\} \supseteq \mathbb{P}\left\{V_{\boldsymbol{\omega}}^{(n)} \chi_{\boldsymbol{\Lambda}_{L}^{(n)}(\boldsymbol{x})} \leq \frac{\epsilon}{2}\right\}>0,
$$

where the strict positivity comes from Definition 1.1.

Since $\Sigma^{(n)}=\sigma\left(H_{\omega}^{(n)}\right)$ for P-a.e. $\boldsymbol{\omega}$, we conclude from (A.1) that we have $\operatorname{dist}\left(\lambda, \Sigma^{(n)}\right) \leq \varepsilon$ for all $\varepsilon>0$, and hence $\lambda \in \Sigma^{(n)}$.

\section{B. Unique continuation principle for spectral projections of Schrödinger operators on arbitrary rectangles}

In this appendix we extend [24, Theorems 1.1 and 2.2] to arbitrary rectangles. Let $H=-\Delta+V$ be a Schrödinger operator on $\mathrm{L}^{2}\left(\mathbb{R}^{d}\right)$. Given a rectangle $\Lambda \subset \mathbb{R}^{d}$, let $H_{\Lambda}=-\Delta_{\Lambda}+V_{\Lambda}$ denote the restriction of $H$ to the rectangle $\Lambda$ with either Dirichlet or periodic boundary condition: $\Delta_{\Lambda}$ is the Laplacian with either Dirichlet or periodic boundary condition and $V_{\Lambda}$ is the restriction of $V$ to $\Lambda$. (We will abuse the notation and simply write $V$ for $V_{\Lambda}$, i.e., $H_{\Lambda}=-\Delta_{\Lambda}+V$ on $\mathrm{L}^{2}(\Lambda)$.) By a unique continuation principle for spectral projections (UCPSP) we mean an estimate of the form

$$
\chi_{I}\left(H_{\Lambda}\right) W \chi_{I}\left(H_{\Lambda}\right) \geq \kappa \chi_{I}\left(H_{\Lambda}\right),
$$

where $\chi_{I}$ is the characteristic function of an interval $I \subset \mathbb{R}, W \geq 0$ is a potential, and $\kappa>0$ is a constant.

In this appendix we use the Euclidean norm on $\mathbb{R}^{d}$ :

$$
|x|=|x|_{2}:=\left(\sum_{j=1}^{d}\left|x_{j}\right|^{2}\right)^{\frac{1}{2}} \quad \text { for } x=\left(x_{1}, x_{2}, \ldots, x_{d}\right) \in \mathbb{R}^{d} .
$$


Distances between sets in $\mathbb{R}^{d}$ will be measured with respect to norm $|x|$. The ball centered at $x \in \mathbb{R}^{d}$ with radius $\delta>0$ is given by

$$
B(x, \delta):=\left\{y \in \mathbb{R}^{d} ;|y-x|<\delta\right\} .
$$

We consider rectangles

$$
\Lambda=\Lambda_{\boldsymbol{L}}(a)=a+\prod_{j=1}^{d}\left(-\frac{L_{j}}{2}, \frac{L_{j}}{2}\right)=\prod_{j=1}^{d}\left(a_{j}-\frac{L_{j}}{2}, a_{j}+\frac{L_{j}}{2}\right),
$$

where $a \in \mathbb{R}^{d}$ and $\boldsymbol{L}=\left(L_{1}, \ldots, L_{d}\right) \in(0, \infty)^{d}$. The box $\Lambda_{L}(x)=x+\left(-\frac{L}{2}, \frac{L}{2}\right)^{d}$ centered at $x \in \mathbb{R}^{d}$ with side of length $L$ is the special case $L_{1}=\cdots=L_{d}=L$. Given a rectangle $\Lambda$ we set

$$
\widehat{\Lambda}=\Lambda \cap \mathbb{Z}^{d} \quad \text { and } \quad \hat{\Lambda}=\left\{k \in \widehat{\Lambda} ; \Lambda_{1}(k) \subset \Lambda\right\} .
$$

$H_{\Lambda}$ will denote the restriction of $H$ to the rectangle $\Lambda$ with either Dirichlet or periodic boundary condition.

Given subsets $A$ and $B$ of $\mathbb{R}^{d}$, and a function $\varphi$ on the set $B$, we set

$$
\varphi_{A}:=\varphi \chi_{A \cap B} .
$$

In particular, given $x \in \mathbb{R}^{d}$ and $\delta>0$ we write

$$
\varphi_{x, \delta}:=\varphi_{B(x, \delta)} .
$$

We let $\mathbb{N}_{\text {odd }}$ denote the set of odd natural numbers. If $K$ is an operator on a Hilbert space, $\mathcal{D}(K)$ will denote its domain. By a constant we will always mean a finite constant. We will use $C_{a, b, \ldots}, C_{a, b, \ldots}^{\prime}, C(a, b, \ldots)$, etc., to denote a constant depending only on the parameters $a, b, \ldots$.

The following is an extension of [24, Theorem 1.1] to rectangles with arbitrary centers and side lengths.

Theorem B.1. Let $H=-\Delta+V$ be a Schrödinger operator on $\mathrm{L}^{2}\left(\mathbb{R}^{d}\right)$, where $V$ is a bounded potential. Fix $\delta \in\left(0, \frac{1}{2}\right]$, let $\left\{y_{k}\right\}_{k \in \mathbb{Z}^{d}}$ be sites in $\mathbb{R}^{d}$ with $B\left(y_{k}, \delta\right) \subset$ $\Lambda_{1}(k)$ for all $k \in \mathbb{Z}^{d}$. Given $E_{0}>0$, set $K=K\left(V, E_{0}\right)=2\|V\|_{\infty}+E_{0}$. Consider a rectangle $\Lambda$ as in (B.2), where $a \in \mathbb{R}^{d}$ and $L_{j} \geq 114 \sqrt{d}$ for $j=1, \ldots, d$, and set

$$
W^{(\Lambda)}=\sum_{k \in \hat{\hat{\Lambda}}} \chi_{B\left(y_{k}, \delta\right)} .
$$


There exists a constant $M_{d}>0$, such that, defining $\gamma=\gamma(d, K, \delta)>0$ by

$$
\gamma^{2}=\frac{1}{2} \delta^{M_{d}\left(1+K^{\frac{2}{3}}\right)},
$$

then for any closed interval $I \subset\left(-\infty, E_{0}\right]$ with $|I| \leq 2 \gamma$ we have

$$
\chi_{I}\left(H_{\Lambda}\right) W^{(\Lambda)} \chi_{I}\left(H_{\Lambda}\right) \geq \gamma^{2} \chi_{I}\left(H_{\Lambda}\right) .
$$

Remark B.2. It follows, using Theorem B.1 in the proofs, that the optimal Wegner estimates for (one-particle) crooked Anderson Hamiltonians given in [24, Theorems 1.4 and 1.5] hold for a rectangle $\Lambda$ as in (B.2), where $a \in \mathbb{R}^{d}$ and $L_{j} \geq 114 \sqrt{d}+\delta_{+}$for $j=1, \ldots, d$. (In particular, they hold on arbitrary boxes $\Lambda=\Lambda_{L}\left(x_{0}\right)$, where $x_{0} \in \mathbb{R}^{d}$ and $L \geq 114 \sqrt{d}+\delta_{+}$.)

For convenience we recall the quantitative unique continuation principle [4, Theorem 3.2] as stated in [24, Theorem 2.1].

Theorem B.3. Let $\Omega$ be an open subset of $\mathbb{R}^{d}$ and consider a real measurable function $V$ on $\Omega$ with $\|V\|_{\infty} \leq K<\infty$. Let $\psi \in \mathrm{H}^{2}(\Omega)$ be real valued and let $\zeta \in \mathrm{L}^{2}(\Omega)$ be defined by

$$
-\Delta \psi+V \psi=\zeta \text { a.e. on } \Omega .
$$

Let $\Theta \subset \Omega$ be a bounded measurable set where $\left\|\psi_{\Theta}\right\|_{2}>0$. Set

$$
Q(x, \Theta):=\sup _{y \in \Theta}|y-x| \quad \text { for } x \in \Omega .
$$

Consider $x_{0} \in \Omega \backslash \bar{\Theta}$ such that

$$
Q=Q\left(x_{0}, \Theta\right) \geq 1 \quad \text { and } \quad B\left(x_{0}, 6 Q+2\right) \subset \Omega .
$$

Then, given

$$
0<\delta \leq \min \left\{\operatorname{dist}\left(x_{0}, \Theta\right), \frac{1}{2}\right\},
$$

we have

$$
\left(\frac{\delta}{Q}\right)^{m_{d}\left(1+K^{\frac{2}{3}}\right)\left(Q^{\frac{4}{3}}+\log \frac{\left\|\psi_{\Omega}\right\|_{2}}{\left\|\psi_{\Theta}\right\|_{2}}\right)}\left\|\psi_{\Theta}\right\|_{2}^{2} \leq\left\|\psi_{x_{0}, \delta}\right\|_{2}^{2}+\delta^{2}\left\|\zeta_{\Omega}\right\|_{2}^{2},
$$

where $m_{d}>0$ is a constant depending only on $d$.

The following theorem is a version of [24, Theorem 2.2] for rectangles with arbitrary centers and side lengths. 
Theorem B.4. Let $H=-\Delta+V$ be a Schrödinger operator on $\mathrm{L}^{2}\left(\mathbb{R}^{d}\right)$, where $V$ is a bounded potential with $\|V\|_{\infty} \leq K$. Fix $\delta \in\left(0, \frac{1}{2}\right]$, let $\left\{y_{k}\right\}_{k \in \mathbb{Z}^{d}}$ be sites in $\mathbb{R}^{d}$ with $B\left(y_{k}, \delta\right) \subset \Lambda_{1}(k)$ for all $k \in \mathbb{Z}^{d}$. Consider a rectangle $\Lambda$ as in (B.2), where $a \in \mathbb{R}^{d}$ and $L_{j} \geq 114 \sqrt{d}$ for $j=1, \ldots, d$. Then for all real-valued $\psi \in \mathcal{D}\left(\Delta_{\Lambda}\right)$ we have

$$
\delta^{M_{d}\left(1+K^{\frac{2}{3}}\right)}\left\|\psi_{\Lambda}\right\|_{2}^{2} \leq \sum_{k \in \hat{\hat{\Lambda}}}\left\|\psi_{y_{k}, \delta}\right\|_{2}^{2}+\delta^{2}\left\|((-\Delta+V) \psi)_{\Lambda}\right\|_{2}^{2},
$$

where $M_{d}>0$ is a constant depending only on $d$.

Proof. As in [18, Proof of Corollary A.2], we extend $V$ and functions $\varphi \in \mathrm{L}^{2}(\Lambda)$ to $\mathbb{R}^{d}$.

For Dirichlet boundary condition, given $\varphi \in \mathrm{L}^{2}(\Lambda)$, we extend it to a function $\tilde{\varphi} \in \mathrm{L}_{\mathrm{loc}}^{2}\left(\mathbb{R}^{d}\right)$ by setting $\tilde{\varphi}=\varphi$ on $\Lambda$ and $\tilde{\varphi}=0$ on $\partial \Lambda$, and requiring

$$
\tilde{\varphi}(x)=-\tilde{\varphi}\left(x+\left(L_{j}-2 \theta_{j}\left(x_{j}-a_{j}\right)\right) \mathrm{e}_{j}\right) \quad \text { for all } x \in \mathbb{R}^{d} \text { and } j \in\{1,2 \ldots, d\},
$$

where $\left\{\mathrm{e}_{j}\right\}_{j=1,2 \ldots, d}$ is the canonical orthonormal basis in $\mathbb{R}^{d}$, and for each $t \in \mathbb{R}$ we define $\theta_{j}(t) \in\left(-\frac{L_{j}}{2}, \frac{L_{j}}{2}\right]$ by

$$
t=k L_{j}+\theta_{j}(t)
$$

with $k \in \mathbb{Z}$. We also extend the potential $V$ to a potential $\widehat{V}$ on $\mathbb{R}^{d}$ by by setting $\widehat{V}=V$ on $\Lambda$ and $V=0$ on $\partial \Lambda$, and requiring that, for all $x \in \mathbb{R}^{d}$ and $j \in$ $\{1,2 \ldots, d\}$,

$$
\widehat{V}(x)=\widehat{V}\left(x+\left(L_{j}-2 \theta_{j}\left(x_{j}-a_{j}\right)\right) \mathrm{e}_{j}\right) .
$$

Note that $\|\hat{V}\|_{\infty}=\|V\|_{\infty} \leq K$. Moreover, $\psi \in \mathcal{D}\left(\Delta_{\Lambda}\right)$ implies $\tilde{\psi} \in \mathrm{H}_{\mathrm{loc}}^{2}\left(\mathbb{R}^{d}\right)$ and

$$
\widetilde{(-\Delta+V) \psi}=(-\Delta+\hat{V}) \tilde{\psi} .
$$

For periodic boundary condition, we extend $\varphi \in \mathrm{L}^{2}(\Lambda)$ and $V$ to periodic functions $\tilde{\varphi}$ and $\hat{V}$ on $\mathbb{R}^{d}$ of period $\left(L_{1}, \ldots, L_{d}\right)$; note $\|\hat{V}\|_{\infty}=\|V\|_{\infty} \leq K$. Moreover, $\psi \in \mathcal{D}\left(\Delta_{\Lambda}\right)$ implies $\tilde{\psi} \in \mathrm{H}_{\mathrm{loc}}^{2}\left(\mathbb{R}^{d}\right)$ and we have (B.14).

Let $\tau=\left(\tau_{1}, \ldots, \tau_{d}\right)$ be given by

$$
\tau_{j}=\min \left\{t \geq 2 ; L_{j} \in t \mathbb{N}_{\text {odd }}\right\}=\frac{L_{j}}{2\left\lfloor\frac{L_{j}-2}{4}\right\rfloor+1}, \quad j=1,2, \ldots, d .
$$


It follows that $\left(L_{j}>12 \sqrt{d} \geq 12\right)$

$$
2 \leq \tau_{j} \leq \frac{L_{j}}{2\left(\frac{L_{j}-2}{4}-1\right)+1}=\frac{2}{1-\frac{4}{L_{j}}}<\frac{2}{1-\frac{4}{12}}=3,
$$

so

$$
\tau_{\infty}=\max _{j=1, \ldots, d} \tau_{j}<3
$$

We let $\tau \mathbb{Z}^{d}=\prod_{j=1}^{d} \tau_{j} \mathbb{Z}$ and $\Lambda^{(\tau)}=\left(a+\tau \mathbb{Z}^{d}\right) \cap \Lambda$. Then

$$
\bar{\Lambda}=\bigcup_{\kappa \in \Lambda^{(\tau)}} \overline{\Lambda_{\tau}(\kappa)}
$$

We define

$$
J: \Lambda^{(\tau)} \longrightarrow \widehat{\hat{\Lambda}}
$$

in such a way that

$$
\Lambda_{1}(\mathcal{J}(\kappa)) \subset \Lambda_{\tau}(\kappa) \text { for all } \kappa \in \Lambda^{(\tau)} .
$$

This can always be done since $\tau_{j} \geq 2$ for $j=1, \ldots, d$; note that $\mathcal{J}$ is one to one.

Let $Y \in \mathbb{N}_{\text {odd }}, Y \leq \frac{L_{j}}{6}<\frac{L_{j}}{2 \tau_{j}}$ for $j=1,2, \ldots, d$. It follows that for all $\varphi \in \mathrm{L}^{2}(\Lambda)$ we have (see [29, Subsection 5.2])

$$
\sum_{\kappa \in \Lambda^{(\tau)}}\left\|\tilde{\varphi}_{\Lambda_{Y \tau}(\kappa)}\right\|_{2}^{2} \leq\left(2 \tau_{\infty} Y\right)^{d}\left\|\varphi_{\Lambda}\right\|_{2}^{2} \leq(6 Y)^{d}\left\|\varphi_{\Lambda}\right\|_{2}^{2} .
$$

We now fix $\psi \in \mathcal{D}\left(\Delta_{\Lambda}\right)$. Following Rojas-Molina and Veselić, we call a site $\kappa \in \Lambda^{(\tau)}$ dominating (for $\psi$ ) if

$$
\left\|\psi_{\left.\Lambda_{\boldsymbol{\tau}}(\kappa)\right)}\right\|_{2}^{2} \geq \frac{1}{2(6 Y)^{d}}\left\|\tilde{\psi}_{\Lambda_{Y \tau}(\kappa)}\right\|_{2}^{2}
$$

Letting $\hat{D} \subset \Lambda^{(\tau)}$ denote the collection of dominating sites, Rojas-Molina and Veselić [29, Subsection 5.2] observed that it follows from equations (B.18), (B.19), and (B.17), that

$$
\sum_{\kappa \in \hat{D}}\left\|\psi_{\left.\Lambda_{\tau}(\kappa)\right)}\right\|_{2}^{2} \geq \frac{1}{2}\left\|\psi_{\Lambda}\right\|_{2}^{2}
$$

We define a map

$$
J: \Lambda^{(\tau)} \longrightarrow \Lambda^{(\tau)}
$$

by

$$
J(\kappa)= \begin{cases}\kappa+2 \tau_{1} \mathrm{e}_{1} & \text { if } \kappa+2 \tau_{1} \mathrm{e}_{1} \in \Lambda^{(\tau)}, \\ \kappa-2 \tau_{1} \mathrm{e}_{1} & \text { if } \kappa+2 \tau_{1} \mathrm{e}_{1} \notin \Lambda^{(\tau)}\end{cases}
$$


Note that $J$ is well defined, and

$$
\# J^{-1}(\{\kappa\}) \leq 2 \text { for all } \kappa \in \Lambda^{(\tau)} .
$$

We have (see (B.8))

$$
\begin{aligned}
Q\left(y_{\mathcal{J}(J(\kappa))}, \Lambda_{\boldsymbol{\tau}}(\kappa)\right) & =\sup _{x \in \Lambda_{\tau}(\kappa)}\left|x-y_{\mathcal{\jmath}(J(\kappa))}\right| \\
& \leq \sup _{x \in \Lambda_{\tau}(\kappa)}|x-\kappa|+|\kappa-J(\kappa)|+\left|J(\kappa)-y_{\mathcal{J}(J(\kappa))}\right| \\
& \leq \frac{\tau_{\infty}}{2} \sqrt{d}+2 \tau_{1}+\frac{\tau_{\infty}}{2} \sqrt{d} \\
& \leq 3 \tau_{\infty} \sqrt{d} \\
& \leq 9 \sqrt{d} .
\end{aligned}
$$

for all $k \in \Lambda^{(\tau)}$.

For each $\kappa \in \Lambda^{(\tau)}$ we will apply Theorem B.3 with $\Omega=\Lambda_{Y \tau}(\kappa), \Theta=\Lambda_{\tau}(\kappa)$, and $x_{0}=y_{\mathcal{\partial}(J(\kappa))}$. We need to guarantee (B.9), that is,

$$
B\left(y_{\mathcal{J}(J(\kappa))}, 6 Q\left(y_{\mathcal{\partial}(J(\kappa))}, \Lambda_{\tau}(\kappa)\right)+2\right) \subset \Lambda_{Y \tau}(\kappa) \text { for all } k \in \hat{D} .
$$

It suffices, using (B.23) and $\tau_{j} \geq 2$, to have

$$
\left|y_{\mathcal{\partial}(J(\kappa))}-\kappa\right|+9 \sqrt{d} \leq \frac{\tau_{\infty}}{2} \sqrt{d}+2 \tau_{1}+9 \sqrt{d} \leq \frac{33}{2} \sqrt{d} \leq \frac{2 Y}{2}=Y .
$$

We thus choose

$$
Y=\min \left\{n \in \mathbb{N}_{\text {odd }} ; n \geq \frac{33}{2} \sqrt{d}\right\} \leq \frac{33}{2} \sqrt{d}+2 \leq 19 \sqrt{d} .
$$

Since we want $Y \leq \frac{L_{j}}{6}<\frac{L_{j}}{2 \tau_{j}}$ for $j=1,2, \ldots, d$, we require $L_{j} \geq 114 \sqrt{d}$ for $j=1,2, \ldots, d$.

Applying Theorem B.3, for each $\kappa \in \Lambda^{(\tau)}$ we get

$$
\delta^{m_{d}^{\prime}\left(1+K^{\left.\frac{2}{3}\right)}\right.}\left\|\psi_{\Lambda_{\tau}(\kappa)}\right\|_{2}^{2} \leq\left\|\psi_{y_{\mathcal{J}(J(\kappa))}, \delta}\right\|_{2}^{2}+\delta^{2}\left\|\tilde{\zeta}_{\Lambda_{Y \tau}(\kappa)}\right\|_{2}^{2},
$$

where $\zeta=(-\Delta+V) \psi$ and $m_{d}^{\prime}>0$ is a constant depending only on $d$. Summing over $\kappa \in \hat{D}$ and using (B.20), (B.22), (B.18), and (B.24), and we get

$$
\begin{aligned}
\frac{1}{2} \delta^{m_{d}^{\prime}\left(1+K^{\frac{2}{3}}\right)}\left\|\psi_{\Lambda}\right\|_{2}^{2} & \leq 2 \sum_{k \in \hat{\hat{\Lambda}}}\left\|\psi_{y_{k}, \delta}\right\|_{2}^{2}+(6 Y)^{d} \delta^{2}\left\|\zeta_{\Lambda}\right\|_{2}^{2} \\
& \leq 2 \sum_{k \in \hat{\hat{\Lambda}}}\left\|\psi_{y_{k}, \delta}\right\|_{2}^{2}+(114 \sqrt{d})^{d} \delta^{2}\left\|\zeta_{\Lambda}\right\|_{2}^{2},
\end{aligned}
$$

so (B.12) follows. 
Proof of Theorem B.1. Given $E_{0}>0$, set $K=K\left(V, E_{0}\right)=2\|V\|_{\infty}+E_{0}$, and let $\gamma$ be given by (B.5), where $M_{d}>0$ is the constant in Theorem B.4. Let $I \subset\left(-\infty, E_{0}\right]$ be a closed interval with $|I| \leq 2 \gamma$. Since $\sigma\left(H_{\Lambda}\right) \subset\left[-\|V\|_{\infty}, \infty\right)$ for any rectangle $\Lambda$, without loss of generality we assume $I=[E-\gamma, E+\gamma]$ with $E \in\left[-\|V\|_{\infty}, E_{0}\right]$, so

$$
\|V-E\|_{\infty} \leq\|V\|_{\infty}+\max \left\{E_{0},\|V\|_{\infty}\right\} \leq K .
$$

Moreover, for any rectangle $\Lambda$ we have

$$
\left\|\left(H_{\Lambda}-E\right) \psi\right\|_{2} \leq \gamma\|\psi\|_{2} \quad \text { for all } \psi \in \operatorname{Ran} \chi_{I}\left(H_{\Lambda}\right) .
$$

Let $\Lambda$ be a rectangle as in Theorem B.4 and $\psi \in \operatorname{Ran} \chi_{I}\left(H_{\Lambda}\right)$. If $\psi$ is realvalued, it follows from Theorem B.4, (B.5), and (B.26) that

$$
2 \gamma^{2}\|\psi\|_{2}^{2} \leq \sum_{k \in \hat{\hat{\Lambda}}}\left\|\psi_{y_{k}, \delta}\right\|_{2}^{2}+\gamma^{2}\|\psi\|_{2}^{2},
$$

yielding

$$
\gamma^{2}\|\psi\|_{2}^{2} \leq \sum_{k \in \hat{\hat{\Lambda}}}\left\|\psi_{y_{k}, \delta}\right\|_{2}^{2}=\left\|W^{(\Lambda)} \psi\right\|_{2}^{2},
$$

where the equality follows from (B.4). For arbitrary $\psi \in \operatorname{Ran} \chi_{I}\left(H_{\Lambda}\right)$, we write $\psi=\Re \psi+i \Im \psi$, and note that $\Re \psi, \Im \psi \in \operatorname{Ran} \chi_{I}\left(H_{\Lambda}\right),\|\psi\|_{2}^{2}=\|\Re \psi\|_{2}^{2}+\|\Im \psi\|_{2}^{2}$, and, since $W^{(\Lambda)}$ is real-valued, $\left\|W^{(\Lambda)} \psi\right\|_{2}^{2}=\left\|W^{(\Lambda)} \Re \psi\right\|_{2}^{2}+\left\|W^{(\Lambda)} \Im \psi\right\|_{2}^{2}$. Recalling $\left(W^{(\Lambda)}\right)^{2}=W^{(\Lambda)}$, we conclude that

$$
\gamma^{2}\langle\psi, \psi\rangle=\gamma^{2}\|\psi\|_{2}^{2} \leq\left\|W^{(\Lambda)} \psi\right\|_{2}^{2}=\left\langle\psi, W^{(\Lambda)} \psi\right\rangle,
$$

for all $\psi \in \operatorname{Ran} \chi_{I}\left(H_{\Lambda}\right)$, proving (B.6).

\section{References}

[1] M. Aizenman, J. Schenker, R. Friedrich, and D. Hundertmark, Finite volume fractional-moment criteria for Anderson localization. Comm. Math. Phys. 224 (2001), no. 1, 219-253. Dedicated to J. L. Lebowitz. MR 1868998 Zbl 1038.82038

[2] M. Aizenman and S. Warzel, Localization bounds for multiparticle systems. Comm. Math. Phys. 290 (2009), no. 3, 903-934. MR 2525644 Zbl 1186.82055

[3] A. Boutet de Monvel, V. Chulaevsky, S. Stollmann, and Y. Suhov, Wegner-type bounds for a multi-particle continuous Anderson model with an alloy-type external potential. J. Stat. Phys. 138 (2010), no. 4-5, 553-566. MR 2594911 Zbl 1187.82056 
[4] J. Bourgain and A. Klein, Bounds on the density of states for Schrödinger operators. Invent. Math. 194 (2013), no. 1, 41-72. MR 3103255 Zbl 06225182

[5] R. Carmona and J. Lacroix, Spectral theory of random Schrödinger operators. Probability and its Applications. Birkhäuser Boston, Boston, MA, 1990. MR 1102675 Zbl 0717.60074

[6] V. Chulaevsky, A. Boutet de Monvel, and Y. Suhov, Dynamical localization for a multi-particle model with an alloy-type external random potential. Nonlinearity $\mathbf{2 4}$ (2011), no. 5, 1451-1472. MR 2785977 Zbl 1222.82046

[7] V. Chulaevsky and Y. Suhov, Wegner bounds for a two particle tight binding model. Comm. Math. Phys. 283 (2008), no. 2, 479-489. MR 2430641 Zbl 1156.82002

[8] V. Chulaevsky and Y. Suhov, Eigenfunctions in a two-particle Anderson tight binding model. Comm. Math. Phys. 289 (2009), no. 2, 701-723. MR 2506767 Zbl 1177.82080

[9] V. Chulaevsky and Y. Suhov, Multi-particle Anderson localisation: induction on the number of particles. Math. Phys. Anal. Geom. 12 (2009), no. 2, 117-139. MR 2497319 Zbl 1166.82003

[10] J. M. Combes and P. D. Hislop, Localization for some continuous, random Hamiltonians in $d$-dimensions. J. Funct. Anal. 124 (1994), no. 1, 149-180. MR 1284608 Zbl 0801.60054

[11] H. von Dreifus and A. Klein, A new proof of localization in the Anderson tight binding model. Comm. Math. Phys. 124 (1989), no. 2, 285-299. MR 1012868 Zbl 0698.60051

[12] J. Fröhlich, F. Martinelli, E. Scoppola, and T. Spencer, Constructive proof of localization in the Anderson tight binding model. Comm. Math. Phys. 101 (1985), no. 1, 21-46. MR 0814541 Zbl 0573.60096

[13] F. Germinet and A. Klein, Bootstrap multiscale analysis and localization in random media. Comm. Math. Phys. 222 (2001), no. 2, 415-448. MR 1859605 Zbl 0982.82030

[14] F. Germinet and A. Klein, Operator kernel estimates for functions of generalized Schrödinger operators. Proc. Amer. Math. Soc. 131 (2003), no. 3, 911-920. MR 1937430 Zbl 1013.81009

[15] F. Germinet and A. Klein, Explicit finite volume criteria for localization in continuous random media and applications. Geom. Funct. Anal. 13 (2003), no. 6, 1201-1238. MR 2033837 Zbl 1086.82008

[16] F. Germinet and A. Klein, A characterization of the Anderson metal-insulator transport transition. Duke Math. J. 124 (2004), no. 2, 309-350. MR 2078370 Zbl 1062.82020

[17] F. Germinet and A. Klein, New characterizations of the region of complete localization for random Schrödinger operators. J. Stat. Phys. 122 (2006), no. 1, 73-94. MR 2203782 Zbl 1127.82031 
[18] F. Germinet and A. Klein, A comprehensive proof of localization for continuous Anderson models with singular random potentials. J. Eur. Math. Soc. (JEMS) 15 (2013), no. 1, 53-143. MR 2998830 Zbl 1267.82066

[19] P. D. Hislop and F. Klopp, Optimal Wegner estimate and the density of states for N-body, interacting Schrodinger operators with random potentials. Preprint 2013. arXiv:1310.6959 [math-ph]

[20] W. Kirsch, An invitation to random Schrödinger operators. With an appendix by F. Klopp. In M. Disertori, W. Kirsch, A. Klein, F. Klopp, and V. Rivasseau, Vincent (eds.) Random Schrödinger operators. Panoramas et Synthèses, 25. Société Mathématique de France, Paris, 2008, 1-119. MR 2509110 MR 2516524 (collection) Zbl 1162.82004 Zbl 1151.35002 (collection)

[21] W. Kirsch, A Wegner estimate for multi-particle random Hamiltonians. Zh. Mat. Fiz. Anal. Geom. 4 (2008), no. 1, 121-127, 203. MR 2404176 Zbl 1154.82014

[22] W. Kirsch and F. Martinelli, On the spectrum of Schrödinger operators with a random potential. Comm. Math. Phys. 85 (1982), no. 3, 329-350. MR 0678150 Zbl 0506.60058

[23] A. Klein, Multiscale analysis and localization of random operators. In M. Disertori, W. Kirsch, A. Klein, F. Klopp, and V. Rivasseau, Vincent (eds.) Random Schrödinger operators. Panoramas et Synthèses, 25. Société Mathématique de France, Paris, 2008, 121-159. MR 2509111 MR 2516524 (collection) Zbl 1187.82058 Zbl 1151.35002 (collection)

[24] A. Klein, Unique continuation principle for spectral projections of Schrödinger operators and optimal Wegner estimates for non-ergodic random Schrödinger operators. Comm. Math. Phys. 323 (2013), no. 3, 1229-1246. MR 3106507 Zbl 1281.47026

[25] A. Klein and A. Koines, A general framework for localization of classical waves. I. Inhomogeneous media and defect eigenmodes. Math. Phys. Anal. Geom. 4 (2001), no. 2, 97-130. MR 1860881 Zbl 0987.35154

[26] A. Klein, A. Koines, and M. Seifert, Generalized eigenfunctions for waves in inhomogeneous media. J. Funct. Anal. 190 (2002), no. 1, 255-291. Special issue dedicated to the memory of I. E. Segal. MR 1895534 Zbl 1043.35097

[27] A. Klein and S. Nguyen, The bootstrap multiscale analysis for the multi-particle Anderson model. J. Stat. Phys. 151 (2013), no. 5, 938-973. MR 3055384 Zbl 1272.82021

[28] F. Klopp and H. Zenk, The integrated density of states for an interacting multiparticle homogeneous model and applications to the Anderson model. Adv. Math. Phys. 2009 (2009), article id. 679827, 15 pp. MR 2500947 Zbl 1203.82063

[29] C. Rojas-Molina and I. Veselić, Scale-free unique continuation estimates and applications to random Schrödinger operators. Comm. Math. Phys. 320 (2013), no. 1, 245-274. MR 3046996 Zbl 1276.47051 
A. Klein and S. T. Nguyen

Received November 24, 2013; revised April 28, 2014

Abel Klein, University of California, Irvine, Department of Mathematics, Irvine, CA 92697-3875, U.S.A.

e-mail: aklein@uci.edu

Son T. Nguyen, Mathematics Department, University of Missouri, Columbia, MO 65211, U.S.A.

e-mail: sondgnguyen1@gmail.com 